DRAFT VERSION MARCH 10, 2022

Preprint typeset using $\mathrm{LAT}_{\mathrm{E}} \mathrm{X}$ style emulateapj v. 12/16/11

\title{
NGC 3105: A YOUNG CLUSTER IN THE OUTER GALAXY ${ }^{1,2}$
}

\author{
T. J. DAVIDGE \\ Dominion Astrophysical Observatory, \\ National Research Council of Canada, 5071 West Saanich Road, \\ Victoria, BC Canada V9E 2E7 \\ tim.davidge@nrc.ca \\ Draft version March 10, 2022
}

\begin{abstract}
Images and spectra of the open cluster NGC 3105 have been obtained with GMOS on Gemini South. The $\left(i^{\prime}, g^{\prime}-i^{\prime}\right)$ color-magnitude diagram (CMD) constructed from these data extends from the brightest cluster members to $g^{\prime} \sim 23$. This is $\sim 4-5 \mathrm{mag}$ fainter than previous CMDs at visible wavelengths and samples cluster members with sub-solar masses. Assuming a half-solar metallicity, comparisons with isochrones yield a distance of $6.6 \pm 0.3 \mathrm{kpc}$. An age of at least 32 Myr is found based on the photometric properties of the brightest stars, coupled with the apparent absence of pre-main sequence stars in the lower regions of the CMD. The luminosity function of stars between 50 and 70 arcsec from the cluster center is consistent with a Chabrier (2003, PASP, 115, 763) lognormal mass function. However, at radii smaller than 50 arcsec there is a higher specific frequency of the most massive main sequence stars than at larger radii. Photometry obtained from archival SPITZER images reveals that some of the brightest stars near NGC 3105 have excess infrared emission, presumably from warm dust envelopes. $\mathrm{H} \alpha$ emission is detected in a few early-type stars in and around the cluster, building upon previous spectroscopic observations that found Be stars near NGC 3105. The equivalent width of the $\mathrm{NaD}$ lines in the spectra of early type stars is consistent with the reddening found from comparisons with isochrones. Stars with $i^{\prime} \sim 18.5$ that fall near the cluster main sequence have a spectral-type $\mathrm{A} 5 \mathrm{~V}$, and a distance modulus that is consistent with that obtained by comparing isochrones with the CMD is found assuming solar neighborhood intrinsic brightnesses for these stars. Subject headings: open clusters and associations: individual (NGC 3105)
\end{abstract}

\section{INTRODUCTION}

Simulations of molecular clouds suggest that stars form in deeply embedded filamentary structures that subsequently collapse and merge to form star clusters and a diffusely distributed component (e.g. Bonnell et al. 2011). Assuming that the majority of stars form in such environments then a census of young stars in clusters and in the so-called 'field' suggests that most clusters must be short-lived (e.g. Lada \& Lada 2003; Silva-Villa \& Larsen 2011; Fall \& Chandar 2012). That stars do not form in isolation is important not only for interpreting the nature and evolution of star clusters but also for understanding the properties of stars in the field. This is because the time that stars stay in close physical proximity in their natal environments may affect the properties of the objects that ultimately migrate into the field, such as the binary fraction (e.g. Parker \& Meyer 2014) and the low mass cut-off of the main sequence (MS; e.g. Johnstone et al. 1998; Adams et al. 2004).

Feedback from massive stars likely plays a key role

\footnotetext{
${ }^{1}$ Based on observations obtained at the Gemini Observatory, which is operated by the Association of Universities for Research in Astronomy, Inc., under a cooperative agreement with the NSF on behalf of the Gemini partnership: the National Science Foundation (United States), the National Research Council (Canada), CONICYT (Chile), Ministério da Ciência, Tecnologia e Inovação (Brazil) and Ministerio de Ciencia, Tecnología e Innovación Productiva (Argentina).

2 This research has made use of the NASA/IPAC Infrared Science Archive, which is operated by the Jet Propulsion Laboratory, California Institute of Technology, under contract with the National Aeronautics and Space Administration.
}

in cluster evolution. Feedback may purge the gas that is the dominant contributor to the total system mass early in a cluster's evolution, thereby altering the gravitational potential so that stars are no longer bound to the cluster. Feedback is also a potential regulator of star formation during embedded phases (e.g. Nakamura \& Li 2014), and may trigger star formation in surrounding areas (e.g. Bik et al. 2014; Getman et al. 2014). Feedback may also provide re-cycled material to a star-forming region if star formation proceeds over timescales that are longer than a few Myr (e.g. Palous et al. 2014).

If feedback from massive stars is a significant influence on cluster evolution then the age distribution of embedded clusters should plunge at a time that corresponds to the lifetime of massive stars. Studies of clusters with minimum masses of at least a few $\times 10^{3} \mathrm{M}_{\odot}$ in the nearby spiral galaxy M83 indicate that the oldest embedded clusters have ages $\sim 6$ Myr (Hollyhead et al. 2015), which is roughly consistent with the lifetime of very massive stars. This age also corresponds to the onset of the red supergiant (RSG) evolutionary phase in stellar systems, and so marks a time when the intensity of the local UV radiation field will drop if very massive stars were initially present.

Factors other than feedback almost certainly also affect cluster evolution. The kinematic state of a cluster at the time of gas expulsion likely plays a significant role in its survivability, in the sense that systems that happen to be in a super-virial state at the epoch of gas expulsion will have a greater chance of surviving than those in a sub-virial state (e.g. Farias et al. 2015). The fate of a 
cluster may also depend on environmental factors such as proximity to massive molecular clouds (e.g. Kruijssen et al. 2012), location in a galaxy (e.g. Silva-Villa et al. 2014), and the morphological characteristics of the host galaxy (e.g. de Grijs et al. 2013).

Having a large initial mass may not be a guarantee that a cluster will survive for more than a few Myr. Rather, the fate of a young cluster may be influenced by local conditions within the natal environment. Stars in massive star-forming regions may form in a range of environments with surface densities that span at least 4 orders of magnitude (e.g. Kuhn et al. 2015), and it is the densest sub-structures that might be expected to have the highest chances of long-term survival. Not all systems contain such sub-structures. For example, the massive star-forming complex W33 lacks compact substructures, and will likely evolve into a loose association (e.g. Messineo et al. 2015).

The star-to-star age dispersion in a cluster is one measure of how long star-forming material is retained, and this can be estimated by measuring the age difference between the youngest pre-MS (PMS) stars and the oldest MS stars. Realistic stellar structure models that span a wide range of masses and evolutionary states are required, and the failure to include key physical processes can skew age dispersion estimates. For example, models presented by Somers \& Pinsonneault (2015) indicate that the failure to account for star spots may cause isochrones to detect an erroneous age dispersion in the CMDs of systems that are actually coeval.

Observations suggest that age dispersions up to a few Myr are common in massive young clusters (e.g. Román-Zúñiga et al. 2015; Zeidler et al. 2015; Kuryavtseva et al. 2012). Such a relatively short star-forming history is consistent with observations that point to a rapid collapse for massive systems (e.g. Banerjee \& Kroupa 2015) and the timescale over which feedback may start to influence cluster evolution. Still, evidence for larger age dispersions has been found in some cases (e.g. De Marchi et al. 2011b; Lim et al. 2013). Environment may also play a role in determining the duration of star formation. In particular, the spread in age in low mass clusters that form in low density environments may be significantly larger than in more massive - and presumably more compact - clusters (Pfalzner et al. 2014).

The Galactic disc contains young clusters in a range of environments, and the investigation of clusters and their immediate surroundings throughout the Galaxy will provide insights into the processes that affect cluster evolution. With $\mathrm{R}_{G C}=11.4 \pm 0.6 \mathrm{kpc}$ (Paunzen et al. 2005), the open cluster NGC 3105 is in the outer regions of the Galactic disc. Previous estimates of the age and distance of NGC 3105 are summarized in Table 1. The age estimates in Table 1 draw on different age diagnostics and wavelengths. The ages measured by Sagar et al. (2001) and Paunzen et al. (2005) are based on the photometric properties of MS turn-off (MSTO) and post-MS stars at visible wavelengths. In contrast, the age estimated by Davidge (2014) is based on the MS cut-off (MSCO) and the properties of the cluster PMS sequence in the $(K, J-K)$ color-magnitude diagram (CMD).

In the present paper, deep photometric and spectroscopic observations of stars in NGC 3105 are discussed. Images obtained with the Gemini Multi-Object
TABLE 1

Previous age and distance estimates of NGC 3105

\begin{tabular}{ccl}
\hline $\begin{array}{c}\text { Age } \\
(\mathrm{Myr})\end{array}$ & $\begin{array}{c}\text { Distance } \\
(\mathrm{kpc})\end{array}$ & Reference \\
\hline $25 \pm 10$ & $9.5 \pm 1.5$ & Sagar et al. (2001) \\
$20 \pm 5$ & $8.5 \pm 1.0$ & Paunzen et al. (2005) \\
$25 \pm 5$ & $6.8 \pm 0.3$ & Davidge (2014) \\
\hline & & \\
\hline
\end{tabular}

Spectrograph (GMOS) on Gemini South are used to construct a CMD that extends from the very brightest cluster members to $g^{\prime} \sim 23$, which is where PMS stars might be expected based on some previous age estimates. If the input physics used in stellar structure models are correct then age diagnostics that cover different wavelengths and a broad range of magnitudes should yield the same age. The range of magnitudes covered with the GMOS data enables an investigation of cluster age using indicators that span a range of masses and evolutionary states.

In addition to investigating the age, distance, and reddening of NGC 3105, the GMOS CMD was also used to select targets for spectroscopic observation at visible and red wavelengths. Spectra were obtained of stars that span a range of brightnesses, and the data are used to investigate the line-of-sight interstellar absorption, search for line emission, and estimate spectral types. This is the first spectroscopic survey of NGC 3105 that includes stars that are fainter than the MSTO. Finally, images of NGC 3105 that were obtained as part of the SPITZER (Werner et al. 2004) GLIMPSE (Benjamin et al. 2003) survey are also examined. These data are used to probe the light profile of the cluster and investigate the mid-IR properties of bright stars in and around NGC 3105.

Details of the observations and the steps used to remove instrumental signatures from the data are discussed in Section 2. The light profile of NGC 3105 obtained from archival SPITZER images is investigated in Section 3. The photometric measurements obtained from the GMOS and SPITZER images are described in Section 4, and the CMDs and LFs obtained from these data are examined in Sections 5 and 6 . The stellar spectra are discussed in Section 7, while a discussion and summary of the results follows in Section 8.

\section{OBSERVATIONS \& REDUCTIONS}

\subsection{GMOS Imaging}

Images and visible/red spectra were recorded with GMOS (Hook et al. 2004) on Gemini South as part of program GS-2014A-Q-84 (PI: Davidge). The GMOS detector when these data were recorded 3 was a mosaic of three $2048 \times 4068 \mathrm{EEV}$ CCDs. Each $13.5 \mu \mathrm{m}$ square CCD pixel subtended 0.073 arcsec per side on the sky. All images and spectra for this program were recorded with $2 \times 2$ pixel binning.

$g^{\prime}$ and $i^{\prime}$ images of NGC 3105 were recorded on the night of UT December 31, 2013. The sky conditions were photometric when the data were recorded. The exposure times and the mean full width half maximum (FWHM) of stellar profiles at each exposure time are summarized in Table 2. Short and long exposures were recorded to broaden the magnitude range over which photometry

\footnotetext{
3 The detector in GMOS has since been changed.
} 
TABLE 2

Summary of GMOS Images

\begin{tabular}{ccc}
\hline Filter & Exposures & $\begin{array}{c}\text { FWHM } \\
\text { (arcsec) }\end{array}$ \\
\hline$g^{\prime}$ & $1 \times 1 \mathrm{sec}$ & 0.7 \\
$i^{\prime}$ & $1 \times 300 \mathrm{sec}$ & 0.7 \\
& $1 \times 1 \mathrm{sec}$ & 0.5 \\
& $5 \times 60 \mathrm{sec}$ & 0.5 \\
\hline
\end{tabular}

could be performed. The long exposure $i^{\prime}$ images were recorded with a five point dither pattern that sampled the corners and center of a $10 \times 10$ arcsec square.

In addition to science data, a series of calibration images that are required to process the science data were also obtained. Exposures that monitor the floating bias level and the static bias pattern of the CCDs ('biases') were recorded at the end of the night. Images that measure variations in sensitivity due to instrument optics and pixel-to-pixel differences in detector sensitivity ('flats'), and interference fringes in $i^{\prime}$ 团 ('fringe frames') were provided by Gemini as part of the data package for this program. The flats were constructed from observations of the twilight sky.

A standard processing sequence for CCD mosaic imaging at visible and red wavelengths was applied to remove instrumental signatures. To start, the outputs from the individual CCDs in the science and calibration images were multiplied by their amplifier gains. The bias frames were subtracted from the gain-corrected images, and the results were then divided by the flats. The fringe frame was scaled to match the long and short exposure times of the $i^{\prime}$ science images, and the result was subtracted from these data. The long exposure $i^{\prime}$ images were registered using the centroids of stars located across the imaged field as reference points, and the results were averaged together to construct a final deep $i^{\prime}$ image. The $g^{\prime}$ and short-exposure $i^{\prime}$ images were aligned with the combined deep $i^{\prime}$ image. This required the application of modest offsets $(\leq 0.1$ arcsec $)$ to adjust for slight shifts in telescope pointing that occur during guiding. The final deep $i^{\prime}$ image is shown in the left hand panel of Figure 1.

\subsection{GMOS Spectra}

Multi-slit spectra of stars in and around NGC 3105 were recorded on the night of April 18, 2014. Target selection was based on (1) location on the sky close to the center of NGC 3105, and (2) location on the $\left(i^{\prime}, g^{\prime}-i^{\prime}\right)$ CMD that was constructed from the GMOS images. Targets were restricted to the middle CCD of the detector mosaic to maximize common star-to-star wavelength coverage. In some cases it was possible to place another source in a slit that sampled a high priority target by making minor adjustments to the slit location, and these additional sources constitute a modest serendipitous sample. In all, spectra were recorded of 35 sources, although the spectrum of one source (\# 22) was found to be an artifact of a nearby bright star, and so is not considered further. The locations and photometric properties of the stars for which spectra were obtained are listed in Table 3.

\footnotetext{
${ }^{4}$ Fringing is not significant in the $g^{\prime}$ images.
}

With the exception of the three stars used to align the focal plane mask (see below), the slits had a length of 6 arcsec and a width of 1 arcsec. The light was dispersed with the R400 grating $\left(400 \mathrm{l} / \mathrm{mm} ; \lambda_{\text {blaze }}=7640 \AA\right)$, and the spectral resolution measured from $\mathrm{CuAr}$ emission lines is $\lambda / \Delta \lambda \sim 860 \mathrm{FWHM}$. This resolution is too low to allow radial velocities that are reliable enough to assess cluster membership to be measured. The seeing, estimated from the width of stellar profiles in the slits, was $\sim 1$ arcsec FWHM.

Three stars were used to align the slit mask on the sky, and $2.4 \times 2.4$ arcsec apertures - rather than slits were cut in the mask for these sources. These stars are among the brightest in the sample, and their light profiles could be examined in short exposure images to check that they are centered in their apertures. While spectra were recorded of the alignment stars, their spectroscopic line profiles differ slightly from those recorded through slits given that their spectral resolution is tied to the seeing disk, rather than a 1 arcsec slit. However, there is no noticeable difference between the line profiles of the alignment stars and other science targets, and so they are considered in concert with the slit spectra.

Four 900 second exposures were recorded, with two having a nominal central wavelength of $6800 \AA$ and the other two a central wavelength of $6850 \AA$. This offset in central wavelength allows the portions of the spectrum that fall on gaps between the CCDs in one wavelength setting to be covered with the other setting. A GG455 filter was used to suppress higher spectroscopic orders, and the combined response of this grating + filter combination results in poor performance at wavelengths $<5000 \AA$.

Given the broad wavelength coverage, the start and end wavelengths for each spectrum depends on the location of the star in the science field, and the sources listed in Table 3 have a common wavelength coverage between $\sim 5300$ and $8400 \AA$. This wavelength interval contains atomic and molecular transitions that are sensitive to metallicity, temperature, and surface gravity. Features that are interstellar in origin are also expected in these spectra given that NGC 3105 is viewed through the Galactic disk plane, with a line-of-sight extinction $\mathrm{A}_{V} \sim 3$ magnitudes. Diffuse Interstellar Bands (DIBs) are clearly evident in the spectra of the brightest objects (Section 7.1).

Flat field frames were recorded through the slit mask at each wavelength setting midway through the observing sequence. $\mathrm{CuAr}$ arcs were recorded through the slit mask at each central wavelength at the end of the night. The flat-field and arc light sources are in the Gemini Facility Calibration Unit.

The reduction of the spectra proceeded as follows. The individual exposures recorded for the science, flatfield, and arc frames were initially processed separately, starting with the multiplication by the gain of the output from each CCD. A series of bias frames, recorded at the end of the night, were median-combined, and the result was subtracted from each exposure. This was followed by the removal of cosmic rays, which were identified by applying a running median filter to highlight features that are sharper than those that result from spectroscopic lines and the seeing disk. The different exposures for 


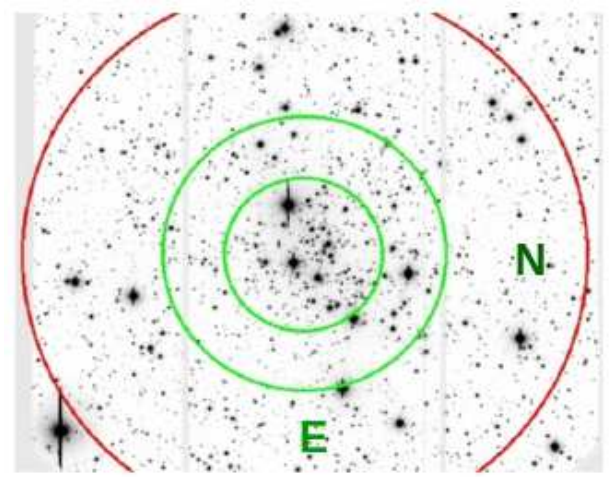

i'

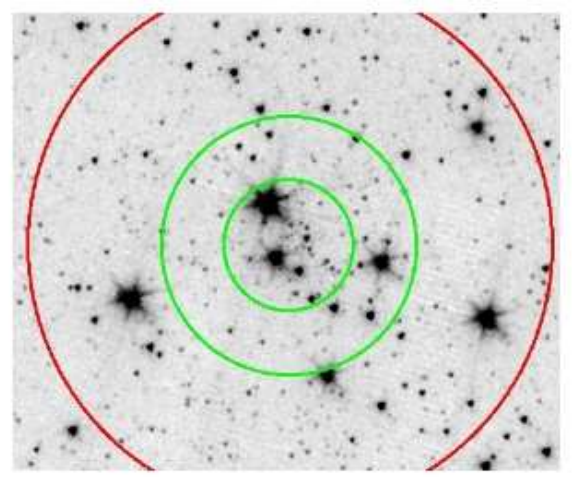

[3.6]

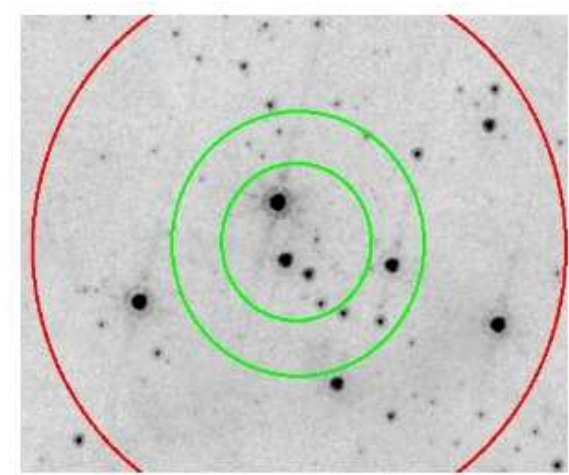

[8.0]

FIG. 1. - Images of NGC 3105 recorded with GMOS and SPITZER. A $5.7 \times 4.6$ arcmin field is shown for each filter. The two vertical stripes in the $i^{\prime}$ image are gaps between the CCDs. The green circles mark the outer boundaries of the Center and Shoulder regions that are used in the analysis of the photometric and spectroscopic data, while the red circle marks the boundary between Fields 1 and 2 .

each observation type (science, flat-field, and arc) were aligned to correct for the wavelength offsets applied during the observing sequence and then averaged together. The null signal in the gaps between CCDs was rejected when computing the mean.

Individual slitlets were extracted from the combined exposures, and each slitlet of a cluster source was divided by the corresponding normalized flat-field spectrum. The flat-fielded cluster spectra were wavelength calibrated using line identifications made from the arcs. The background sky level was measured on a row-by-row basis near the edges of each wavelength calibrated slitlet, and the resulting sky spectrum was subtracted from each of the columns in that slitlet. The sky level for slits containing two stars was measured in the spatial interval where light from the stars was smallest. Spectra of individual stars were extracted from the sky-subtracted slitlets by combining signal within the FWHM of each stellar light profile.

Telluric absorption features are present at wavelengths longward of $6600 \AA$. Spectra of the white dwarf LTT 4364 that were recorded for program GS-2014A-Q12 on May 7, 2014 with the same slit width, grating, airmass, and central wavelength as the NGC 3105 observations were used to suppress these telluric features. The LTT 4364 spectra were processed with the same procedures as the NGC 3105 spectra. Division of the NGC 3105 spectra by the normalized LTT 4364 spectrum corrected well for telluric features, even though NGC 3105 and LTT 4364 were observed on different nights. Division by the LTT 4364 spectrum also removes variations in system response over short and intermediate wavelength intervals, leaving pseudo continuum-corrected spectra that are normalized to the spectral energy distribution (SED) of LTT 4364. The final step in the processing was to fit a continuum function to each spectrum, and divide each telluric-corrected spectrum by the result.

TABLE 3

SPECTROSCOPIC TARGETS

\begin{tabular}{cccccl}
\hline ID & $\begin{array}{c}\text { RA } \\
(2000)\end{array}$ & $\begin{array}{c}\text { Dec } \\
(2000)\end{array}$ & $i^{\prime}$ & $g^{\prime}-i^{\prime}$ & Region $^{\text {ab }}$ \\
& 150.236249 & -54.767349 & 15.823 & 0.955 & $\mathrm{~F}$ \\
2 & 150.232644 & -54.800179 & 18.941 & 1.578 & $\mathrm{~F}$ \\
3 & 150.228896 & -54.806980 & 15.656 & 0.917 & $\mathrm{~F}$ \\
4 & 150.224848 & -54.790791 & 16.473 & 1.212 & $\mathrm{~F}^{*}$ \\
\hline
\end{tabular}

TABLE 3

SPECTROSCOPIC TARGETS

\begin{tabular}{cccccc}
5 & 150.219455 & -54.798790 & 16.305 & 1.200 & $\mathrm{~F}$ \\
6 & 150.215549 & -54.787201 & 18.850 & 1.443 & $\mathrm{~F}$ \\
7 & 150.206852 & -54.789371 & 18.755 & 1.519 & $\mathrm{~F}$ \\
8 & 150.202804 & -54.808510 & 18.731 & 1.473 & $\mathrm{~F}$ \\
9 & 150.199199 & -54.790760 & 18.671 & 1.517 & $\mathrm{~S}$ \\
10 & 150.195293 & -54.784309 & 20.509 & 2.310 & $\mathrm{~S}$ \\
11 & 150.193750 & -54.784167 & 18.576 & 2.018 & $\mathrm{~S}$ \\
12 & 150.191846 & -54.792011 & 20.532 & 2.058 & $\mathrm{~S}$ \\
13 & 150.188556 & -54.800850 & 20.637 & 2.551 & $\mathrm{~S}$ \\
14 & 150.184951 & -54.813831 & 16.347 & 1.128 & $\mathrm{~F}$ \\
15 & 150.181503 & -54.808170 & 18.467 & 1.452 & $\mathrm{~F}$ \\
16 & 150.177312 & -54.799061 & 20.553 & 2.114 & $\mathrm{C}$ \\
17 & 150.172648 & -54.784229 & 20.553 & 2.210 & $\mathrm{C}$ \\
18 & 150.166197 & -54.797981 & 20.486 & 1.974 & $\mathrm{C}$ \\
19 & 150.162449 & -54.801311 & 20.560 & 2.422 & $\mathrm{~S}$ \\
20 & 150.158844 & -54.792580 & 20.499 & 2.205 & $\mathrm{C}$ \\
21 & 150.153751 & -54.789532 & 20.560 & 2.113 & $\mathrm{C}$ \\
$23 \mathrm{c}$ & 150.148501 & -54.798698 & 20.572 & 2.165 & $\mathrm{~S}$ \\
24 & 150.145354 & -54.794891 & 18.569 & 1.484 & $\mathrm{~S}$ \\
25 & 150.141006 & -54.789539 & 20.462 & 2.269 & $\mathrm{~S}$ \\
26 & 150.136042 & -54.795441 & 20.473 & 2.138 & $\mathrm{~F}$ \\
27 & 150.135417 & -54.795417 & 21.148 & 2.566 & $\mathrm{~F}$ \\
28 & 150.126901 & -54.783588 & 16.024 & 0.968 & $\mathrm{~F}$ \\
29 & 150.117602 & -54.784351 & 15.980 & 1.171 & $\mathrm{~F} *$ \\
30 & 150.107846 & -54.801510 & 15.844 & 0.911 & $\mathrm{~F}$ \\
31 & 150.103812 & -54.774899 & 16.240 & 1.180 & $\mathrm{~F} *$ \\
32 & 150.099167 & -54.801750 & 15.378 & 2.632 & $\mathrm{~F}$ \\
33 & 150.098405 & -54.801609 & 16.113 & 1.014 & $\mathrm{~F}$ \\
34 & 150.094800 & -54.812679 & 18.582 & 1.333 & $\mathrm{~F}$ \\
35 & 150.091352 & -54.787239 & 15.948 & 1.018 & $\mathrm{~F}$ \\
\hline & & & & &
\end{tabular}

${ }^{\mathrm{a}} \mathrm{F}=$ Field, $\mathrm{S}=$ Shoulder, $\mathrm{C}=$ Center

b Mask alignment stars indicated with a ' $*$ '

c There is no entry for Star 22 as it was found to be an artifact of a bright star.

\subsection{SPITZER Imaging}

Images recorded with the SPITZER IRAC as part of the GLIMPSE survey (Benjamin et al. 2003) are used in this study to examine the light distribution of NGC 3105 at mid-infrared (MIR) wavelengths (Section 3), and also to investigate the MIR photometric properties of the brightest stars. Post Basic Calibrated Data ('PBCD') images of NGC 3105 and its surroundings were downloaded from the Spitzer Science Archive 5. The PBCD

\footnotetext{
${ }^{5}$ http://irsa.ipac.caltech.edu/data/SPITZER/GLIMPSE/
} 
mosaics have 0.6 arcsec pixel ${ }^{-1}$ sampling, with an angular resolution of $\sim 1.7$ arcsec FWHM in [3.6] and [4.5] (Fazio et al. 2004). The effective exposure time is 2 seconds per pixel.

\section{LIGHT PROFILES OF NGC 3105}

The extremes of the IRAC wavelength coverage are plumbed by the [3.6] and [8.0] filters, which probe different sources of astrophysical emission. The signal at the short wavelength end of the IRAC coverage is predominantly stellar in origin, while the signal in [8.0] may contain a large contribution from interstellar PAH emission, which has a strong feature near $7.6 \mu \mathrm{m}$ and so falls in the [8.0] pass band. The portions of the [3.6] and [8.0] images that sample the region near NGC 3105 are shown in Figure 1. While not obvious from Figure 1, there is patchy emission in and around NGC 3105 in the [8.0] PBCD mosaic, with locally strong emission East of the cluster.

Efforts to trace the light profile of NGC 3105 outside of its central regions are hindered by stochastic effects and contamination from field stars. The S/N ratio of the cluster light profile can be boosted by azimuthally averaging the signal after assuming a shape and orientation for the cluster. The IRAC images of NGC 3105 were binned $12.6 \times 12.6$ arcsec to suppress individual bright stars, and the results were divided into 24 azimuthal zones centered on the cluster. The azimuthal slices were aligned, and the median signal at each pixel location found. Pixels that contain field stars - which are randomly distributed - will be rejected when taking the median, while signal from the cluster light will reinforce constructively. The light profile was then extracted from the median pixel values. This procedure assumes circular isophotes.

The [3.6] and [8.0] light profiles are compared in the top panel of Figure 2. The cluster center was assumed to be at $(\delta, \mathrm{RA})=(-54: 47: 17.6,10: 00: 40.1 ; \mathrm{E} 2000)$, which is near the middle of an asterism defined by four bright, centrally-concentrated stars. The PBCD images cover $\sim 0.9$ degrees $^{2}$, allowing background light levels to be measured at large distances from the cluster. Still, there is uncertainty in the [8.0] background level because of wide-spread, non-uniform emission throughout the sampled area.

Light from NGC 3105 is traced out to at least 100 arcsec in [3.6], and the FWHM of the light profile is $\sim 50$ arcsec. The localized peak in the [3.6] profile near $r \sim 25$ arcsec is residual signal from the bright stars that surround the cluster center (Figure 1). It can be seen in Figure 1 that NGC 3105 is not as obvious in [8.0] as it is in $i^{\prime}$ or [3.6]. In fact, the [8.0] light profile in Figure 2 is much flatter than the [3.6] profile, with a FWHM $\sim 250$ arcsec. The shape of the [8.0] profile follows that of the [3.6] profile at radii $<30$ arcsec, suggesting that the light in [8.0] at these radii is dominated by stars in NGC 3105, rather than PAH emission.

Images in [4.5] and [5.8] were also recorded as part of the GLIMPSE survey. The NGC 3105 light profile in [4.5] - which is not shown here - is almost identical to the [3.6] profile. The light distribution in [5.8] - also not shown here - has a width that is intermediate between those in [3.6] and [8.0]. There is a PAH emission feature near $6.2 \mu \mathrm{m}$ that falls within the [5.8] pass band.
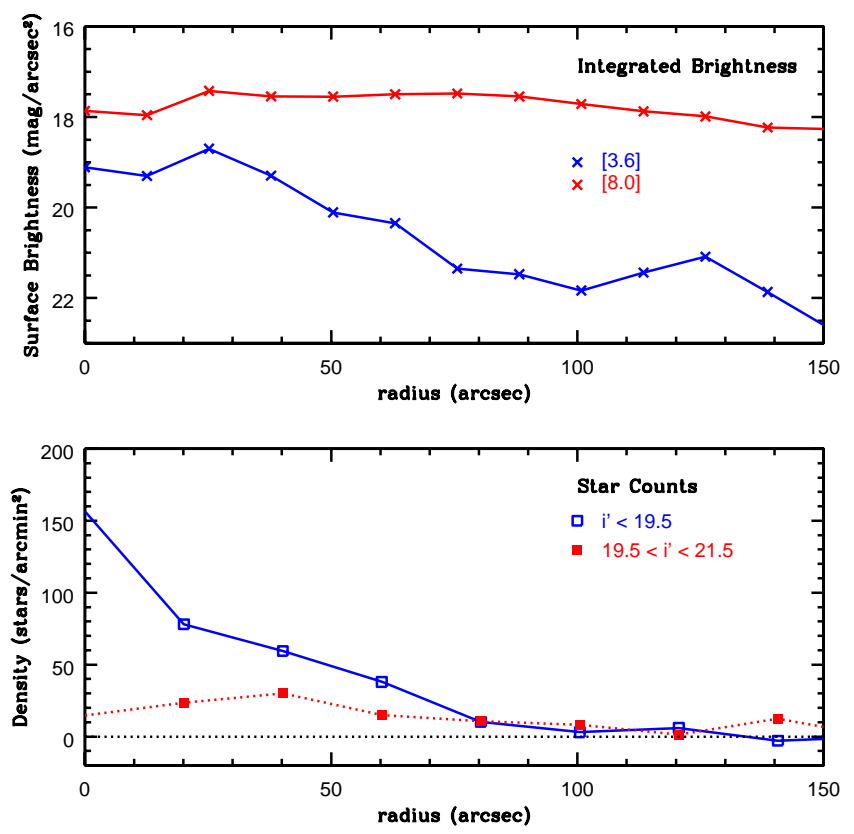

FIG. 2.- (Top panel:) The [3.6] (blue) and [8.0] (red) light profiles of NGC 3105. Background sky levels were measured at large angular offsets from the cluster center. The peak in surface brightness near 25 arcsec is an artifact of the constellation of bright stars that surround the cluster center. The [8.0] light profile is flatter than the [3.6] light profile, and this is attributed to interstellar PAH emission, although cluster stars also contribute to the signal. (Lower panel:) Number counts of stars with $i^{\prime}<19.5$ (open blue squares) and $i^{\prime}$ between 19.5 and 21.5 (filled red squares) made in 20 arcsec wide annuli. Contamination from field stars has been corrected by subtracting star counts in Field 2 (Section 5.1), which samples distances $>177$ arcsec from the cluster center. Stars with $i^{\prime}>19.5$ are more centrally concentrated than the stars in the fainter sample.

Light profiles were also constructed from images recorded as part of the Wide-field Infrared Survey Explorer (WISE; Wright et al. 2010) Allsky survey. Images in the W1 $\left(\lambda_{c e n} \sim 3.4 \mu \mathrm{m}\right)$ and W3 $\left(\lambda_{c e n} \sim 12 \mu \mathrm{m}\right)$ filters were downloaded from the IPAC archive 6. The images were azimuthally smoothed using the procedure described above, and the light profiles extracted. As with the IRAC data, the W1 and W3 light profiles are very different, with the W3 profile being much broader than the $\mathrm{W} 1$ profile. The $\mathrm{W} 3$ bandpass samples PAH emission features.

\section{PHOTOMETRIC MEASUREMENTS}

Stellar photometry was done with the point spread function (PSF)-fitting routine ALLSTAR (Stetson \& Harris 1988). Tasks in the DAOPHOT package (Stetson 1987) were used to generate the source catalogues, preliminary brightnesses, and PSFs that serve as input to ALLSTAR. The PSFs were constructed from stars that are bright, isolated, and unsaturated. Contamination from faint stars close to the PSF stars was removed iteratively, using progressively improved versions of the PSF to subtract out these objects.

The photometric measurements for all but the brightest stars in the GMOS images were made by fitting the PSF within the FWHM of the stellar profiles.

${ }^{6}$ http://irsa.ipac.caltech.edu/Missions/wise.html 
However, the bright MS and evolved stars in NGC 3105 that are important for estimating age are saturated in the short $i^{\prime}$ exposures. While photoelectric magnitudes have been published for the brightest stars in and around NGC 3105 (Moffat \& Fitzgerald 1974; Fitzgerald et al. 1977), these are not in the photometric system used here. To recover the brightnesses of these stars, ALLSTAR was run a second time on the short exposure $i^{\prime}$ image, but this time with the PSF fit over a much larger portion of the stellar profile than just the central regions. The central, saturated parts of the stellar profiles are rejected in the fitting process, so that profile fitting is done only in the unsaturated outer regions of the PSF.

The IRAC photometry was calibrated using zeropoints from Reach et al. (2005). Zeropoints for the GMOS photometry were obtained from observations of standard stars that were recorded on January 12014 . The standard stars are in the 075944-59550 field, and their magnitudes were taken from the Southern Standard Stars for the u'g'r'i'z' System website 7. The zeropoints obtained from these observations fall within the range of values archived for this instrument 8 .

\section{COLOR-MAGNITUDE DIAGRAMS}

\subsection{GMOS CMD}

$\left(i^{\prime}, g^{\prime}-i^{\prime}\right)$ CMDs of the area in and around NGC 3105 are shown in Figure 3. The photometry for objects with $i^{\prime}<17.5$ is from the short exposure images, while the photometry of fainter objects is from the long exposure images. The photometric measurements for stars with $i^{\prime}>12.5$, which are saturated in the short $i^{\prime}$ exposures, were made by fitting the PSF to the wings of the stellar profiles (Section 4). The general appearance of the bright end of the CMD is similar to that in previously published CMDs of this cluster.

CMDs are shown for stars in four different regions, with the boundaries defined in part using the [3.6] light profile in Figure 2. The outer radius of the Center region is at the HWHM of the [3.6] light profile, while the outer radius of the Shoulder region is where the [3.6] light profile flattens. The CMDs in the two remaining panels sample areas that are dominated by field stars, although a modest cluster population is likely present in Field 1 (Section 6). Field 2 was selected to sample the corners of the GMOS science field, which are the areas on the GMOS detector that are furthest from the cluster center. For the remainder of the paper it is assumed that Field 2 is free of cluster stars, and so this area is used to monitor background/foreground contamination.

The radial frequency of cluster stars is investigated in the lower part of Figure 2, where the radial distributions of objects with $i^{\prime} \leq 19.5$ and $i^{\prime}$ between 19.5 and 21.5 , counted in 20 arcsec wide annuli, are compared. Star counts made in Field 2 were subtracted from the number counts to correct for non-cluster stars. There is a clear tendency for objects in the brighter sample to be concentrated within $\sim 80$ arcsec of the cluster center. There is also a tendency for stars in the faint sample to be located within $\sim 100$ arcsec of the cluster center, although there is not a central cusp. For comparison, Mof-

\footnotetext{
7 http://www-star.fnal.gov/

http://www.gemini.edu/sciops/instruments/performancemonitoring/data-products/gmos-n-and-s/photometric-zero-points
}

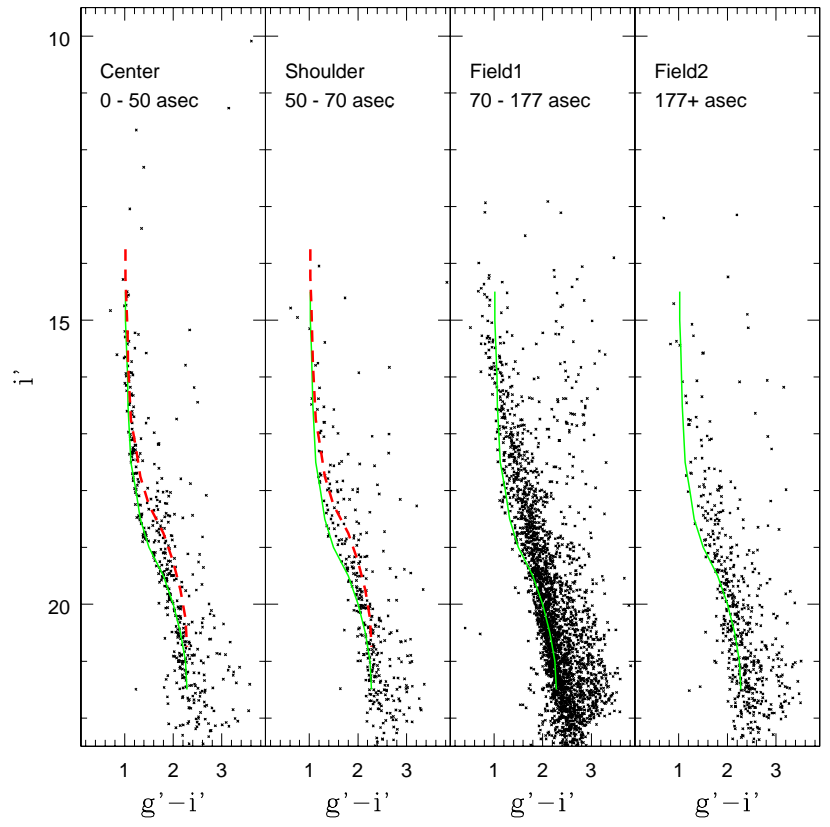

FIG. 3.- The $\left(i^{\prime}, g^{\prime}-i^{\prime}\right)$ CMDs of radial intervals centered on NGC 3105, with boundaries defined in part using the [3.6] light profile (see text). The solid green line is a hand drawn fit to the cluster sequence in the Center field, while the dashed red line is this sequence shifted to brighter values by 0.75 magnitudes to mark the expected location of unresolved equal mass binaries. These are the deepest visible/red CMDs yet published for NGC 3105, and the cluster sequence can be traced to $i^{\prime}>21\left(g^{\prime} \geq 23.5\right)$ in the Center and Shoulder CMDs. The stellar content of Field 2 is used throughout the paper to assess contamination from noncluster objects.

fat \& Fitzgerald (1974) found that a significant number of cluster stars with $B \leq 17.2$ are detected out to distances of 50 - 80 arcsec from the cluster center, although they also argue that some cluster members - identified as such from their photometric properties - may occur out to radii of $\sim 120+$ arcsec. The star counts shown in the lower panel of Figure 2 suggest that cluster stars are not present in large numbers in Fields 1 and 2.

The green line in Figure 3 is a hand-drawn representation of the cluster sequence in the Center CMD. The cluster sequence can be traced to $i^{\prime} \sim 21$ in the Center and Shoulder regions, which corresponds to $g^{\prime} \sim 23.5$, or $V \sim 22.5-23.0$. For comparison, the faint limits of the Paunzen et al. (2005) and Sagar et al. (2001) CMDs are $V \sim 18$. These are thus the deepest (by many magnitudes) photometric measurements yet obtained of NGC 3105 at visible/red wavelengths. Below, it is shown that observations at this depth can provide constraints on the age of NGC 3105, and also sample a magnitude range where signatures of mass segregation become evident in the cluster (Section 6).

Stars with $i^{\prime}<12.5$ are concentrated in the Center CMD, and the blue stars with $i^{\prime}$ between 11.5 and 14 in the Center CMD are likely upper MS or post-MS objects (see below). There are also two red stars with $i^{\prime}<11.2$. If - as suggested by their location near the cluster center - these are cluster members then they are red supergiants (RSGs).

The Field 1 and Field 2 CMDs are dominated by richly populated sequences with ridgelines that fall red- 
ward of the green cluster fiducial. Fore/background stars in the Field $1 \mathrm{CMD}$ form a near-linear sequence that intersects the NGC 3105 fiducial near $i^{\prime}=16$ and $i^{\prime}=21$. Contamination from non-cluster stars is substantial when $i^{\prime}>20$, although number counts discussed below suggest that solar and sub-solar mass stars associated with NGC 3105 may be present in Field 1. Still, the locus of stars with $i^{\prime}>20$ in the Field 1 CMD falls redward of the sequence defined from the Center CMD.

The dispersion in the cluster sequence near $i^{\prime} \sim$ 16 is $\sigma\left(g^{\prime}-i^{\prime}\right)= \pm 0.06$ magnitudes. If due only to variations in line of sight extinction then this corresponds to $\mathrm{a} \sim \pm 0.04$ magnitude dispersion in $\mathrm{E}(\mathrm{B}-\mathrm{V})$. While the narrow nature of the cluster sequence might also suggest that the incidence of unresolved binaries is small, this may not be the case. The dashed line in Figure 3 shows the cluster fiducial shifted upwards by 0.75 magnitudes to simulate the location of unresolved binaries with a mass ratio $=1$. If present in large numbers then it can be seen from Figure 3 that unresolved equal-mass binaries will form a conspicuous sequence in the cluster CMD between $i^{\prime}$ of 17 and 21 .

While there are stars in the Center and Shoulder region CMDs that are close to the binary sequence, there is a significant contribution from field stars. The number of equal mass binaries in NGC 3105 have been estimated by counting objects within \pm 0.1 magnitudes in $g^{\prime}-i^{\prime}$ of the binary sequence with $i^{\prime}$ between 18 and 19. There are 29 sources in this part of the Center and Shoulder region CMDs, whereas the Field 2 CMD contains 18 sources. The mean densities on the sky are then $6.8 \pm 1.3 \mathrm{arcmin}^{-2}$ in the Center and Shoulder regions and $3.2 \pm 0.8 \mathrm{arcmin}^{-2}$ in Field 2. Assuming that Field 2 contains no cluster stars then the difference in projected densities is significant at the $2.4 \sigma$ level, suggesting that NGC 3105 harbors a population of unresolved, equalmass binaries. Based on the number of stars within \pm 0.1 magnitudes in $g^{\prime}-i^{\prime}$ of the main sequence between $i$ of 18.75 and 19.75 , we estimate a binary frequency of $0.5 \pm 0.2$ for equal mass systems. We caution that single stars in NGC 3105 with higher than average extinction may have photometric properties that have been shifted into the area of the CMD that is occupied by equal mass binaries.

The combined CMD of the Center and Shoulder regions is shown in Figures 4 and 5, where comparisons are made with Padova (Bressan et al. 2012) $\mathrm{Z}=0.020$ (Figure 4) and $\mathrm{Z}=0.008$ (Figure 5) isochrones. The models from which these isochrones were constructed include PMS evolution. The isochrones were positioned to match (1) the blue edge of the upper MS, which provides leverage on reddening, and (2) the bend in the CMD that occurs between $i^{\prime}=18$ and 20, which provides constraints on distance. The reddenings and distance moduli that are found by comparing the isochrones with the observations are listed in each figure. The reddenings found from the $\mathrm{Z}=0.008$ and $\mathrm{Z}=0.020$ isochrones are the same, as the intrinsic colors of hot stars are not sensitive to metallicity. However, the distances differ, with $\mathrm{d}=7.9_{-0.3}^{+0.4} \mathrm{kpc}$ for $\mathrm{Z}=0.020$, and $6.6 \pm 0.3 \mathrm{kpc}$ for $\mathrm{Z}=0.008$. $\mathrm{A} \pm 0.1$ magnitude uncertainty in the distance modulus has been assumed, which was found by perturbing the adopted distance modulus and assessing the agreement between

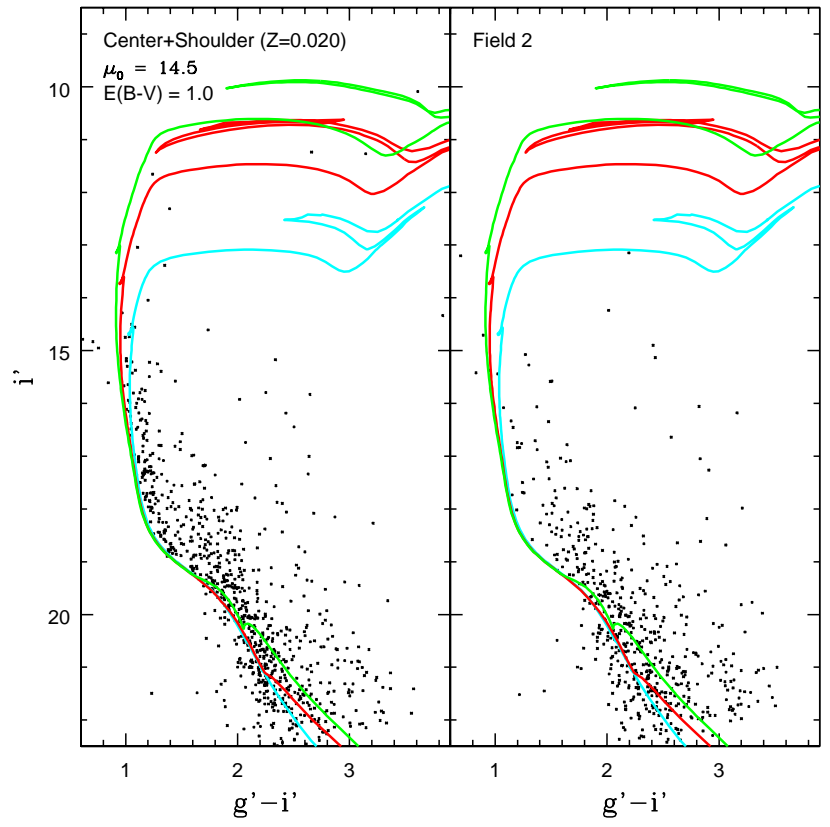

Fig. 4. - Comparisons with $\mathrm{Z}=0.020$ isochrones from Bressan et al. (2012). Sequences with ages $20 \mathrm{Myr}$ (green), $32 \mathrm{Myr}$ (red), and 71 Myr (cyan) are shown. The distance modulus and reddening that result from matching the isochrones to the blue envelope of the cluster sequence are listed in the left hand panel. These models rule out an age as young as $20 \mathrm{Myr}$ based on the color of the cluster sequence at $i^{\prime}>20$, and this is consistent with the photometric properties of what are likely the most evolved cluster members.

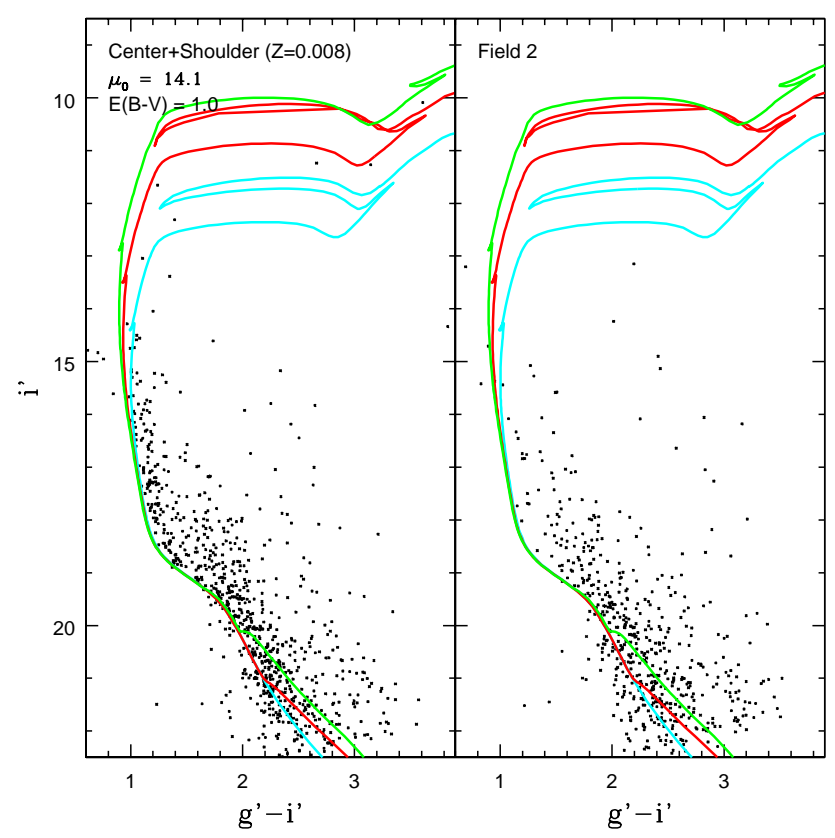

FIG. 5.- Same as Figure 4, but showing comparisons with $\mathrm{Z}=0.008$ isochrones. Isochrones with this metallicity and an age $\sim 32$ Myr match the three brightest evolved stars, while stars with $i^{\prime}$ between 12 and 14 scatter about the 71 Myr model. The CMD at $i^{\prime}>20$ favors an age older than $20 \mathrm{Myr}$.

the models and observations.

The $32 \mathrm{Myr} \mathrm{Z}=0.020$ model best matches the photometric properties of stars with $i^{\prime}>13$, although the 


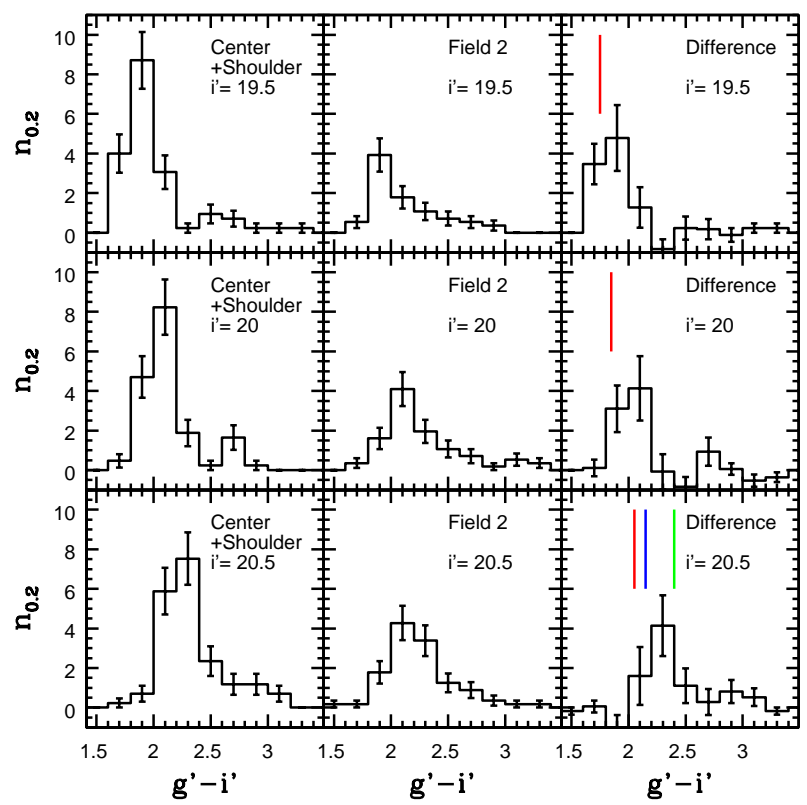

FIG. 6. $-g^{\prime}-i^{\prime}$ distributions in three $i^{\prime}$ intervals. $\mathrm{n}_{0.2}$ is the number of objects per $\operatorname{arcmin}^{2}$ in \pm 0.25 magnitude $i^{\prime}$ intervals with 0.2 magnitude binning in $g^{\prime}-i^{\prime}$. The distributions in the combined Center+Shoulder region are shown in the left hand columns while the color distributions in Field 2, which samples the largest radial distances from the cluster center, are shown in the middle column. The differences between the Center+Shoulder and Field 2 distributions are shown in the right hand panels. The red line indicates the color of the $\mathrm{Z}=0.00832 \mathrm{Myr}$ model at each brightness, while the green and blue lines show the color of 20 and $22 \mathrm{Myr}$ models at $i^{\prime}=20.5$ - the colors of the 20 and $22 \mathrm{Myr}$ isochrones are not indicated in the $i^{\prime}=19.5$ and 20.0 panels as they are the same as the 32 Myr model. These comparisons favor an age for NGC 3105 that is older than $20 \mathrm{Myr}$.

isochrones extend far redward of the three brightest objects in the left hand panel that are presumably the most evolved members of the cluster. As for the faint end of the $\mathrm{CMD}$, the 20 and $32 \mathrm{Myr} \mathrm{Z}=0.020$ models more-orless bracket the main body of stars with $i^{\prime}>20$. The comparisons with the $\mathrm{Z}=0.020$ isochrones thus point to an age $>20$ Myr.

NGC 3105 is in the outer regions of the Galaxy, and so it likely has a sub-solar metallicity. It is thus worth noting that the location of the three candidate RSGs on the CMD fall near the redward extent of the $\mathrm{Z}=0.008$ isochrones. Still, stars with $i^{\prime}$ between 12 and 14 tend to scatter near the $71 \mathrm{Myr}$ isochrone. The 20 Myr sequence falls redward of the main concentration of stars at $i^{\prime}>20$.

The color distributions in the Center+Shoulder and Field 2 CMDs in \pm 0.25 magnitude intervals centered at $i^{\prime}=19.5,20.0$, and 20.5 are compared in Figure 6. A 0.2 magnitude binning in $g^{\prime}-i^{\prime}$ was found to deliver statistically significant numbers of stars in each color interval. The results of subtracting the Field 2 color distribution from that of the Center+Shoulder distribution are shown in the right hand column.

Contamination from non-cluster stars accounts for $\sim 50 \%$ of the objects with $g^{\prime}-i^{\prime}$ between 2 and 2.5. This level of contamination notwithstanding, there remains a statistically significant sample of objects after subtracting the Field 2 color distribution. The colors of 20,22 and
$32 \mathrm{Myr} Z=0.008$ sequences from Bressan et al. (2012) are shown in the right hand column of Figure 6 . All three sequences have the same colors at $i^{\prime}=19.5$ and $i^{\prime}=20$, and so only the $32 \mathrm{Myr}$ model is shown in those panels. The situation changes at $i^{\prime}=20.5$, where the majority of stars fall between the 20 and 32 Myr models. It can be seen from the color of the $22 \mathrm{Myr}$ isochrone that $g^{\prime}-i^{\prime}$ changes quickly with age at this brightness. The persistent tendency for the $32 \mathrm{Myr}$ sequence to fall blueward of the peak in the color distribution at all magnitudes is due to the matching of the isochrones to the blue edge of the cluster sequence in the CMD.

The comparisons in Figure 6 suggest that an age as young as $20 \mathrm{Myr}$ for NGC 3105 can be ruled out, but that an age that is only 2 Myr older would be consistent with the color of the cluster sequence. We note that previous studies have encountered difficulties reproducing the colors of PMS stars at visible and red wavelengths (e.g. Lyra et al. 2006; Bell et al. 2012). Davidge (2015) found an offset $\Delta\left(g^{\prime}-i^{\prime}\right) \sim 0.4$ magnitudes between the observed and model PMS sequence in the $\sim 20$ Myr cluster NGC 2401, in the sense that the cluster sequence is redder than models from Bressan et al. (2012) and Siess et al. (2000). Still, unless there are systematic differences between the properties of PMS stars in NGC 2401 and NGC 3105 (aside from age) then the $g^{\prime}-i^{\prime}$ color of the faint end of the NGC 3105 CMD suggests that the cluster has an age $>20$ Myr. Folding in the properties of the three brightest stars, which are assumed to belong to NGC 3105 and are matched by the $32 \mathrm{Myr}$ isochrones, and considering that the brightest MS stars match the 71 Myr models, we conclude that NGC 3105 has an age of at least 32 Myr.

\subsection{SPITZER CMDs}

Some of the brightest members of NGC 3105 and the surrounding field are shrouded by circumstellar envelopes. One tracer of circumstellar material - $\mathrm{H} \alpha$ emission - is detected among bright early-type stars in and around NGC 3105 (Moffat \& FitzGerald 1974; FitzGerald et al. 1977), and additional emission line sources are discussed in Section 7. Some of the brightest stars in NGC 3105 also may have $\mathrm{Br} \gamma$ in emission (e.g. Figure 6 of Davidge 2014).

If there are thick dust envelopes around some of the stars in NGC 3105 then these objects might also show excess MIR emission. The ([4.5], [3.6] - [4.5]) and ([8.0], [3.6] - [8.0]) CMDs of objects in and around NGC 3105 are shown in Figures 7 and 8. These CMDs cover the same radial intervals as in Figure 3. Only post-MS and bright MS stars in NGC 3105 are sampled by the GLIMPSE observations.

The prominent vertical plume in the SPITZER CMDs is populated by objects that have a range of effective temperatures. While having very different spectraltypes, these objects have similar [3.6]-[4.5] and [3.6]-[8.0] colors (roughly 0 in each case) because the RayleighJeans tail of their SEDs is sampled at these wavelengths. Still, there are two bright objects in the Center field that have redder [3.6]-[8.0] colors than the majority of cluster (and field) members. These are among the brightest cluster members, and their location on the GMOS CMD indicates that they are evolving near the MSTO - hence, they are likely among the most massive stars in NGC 


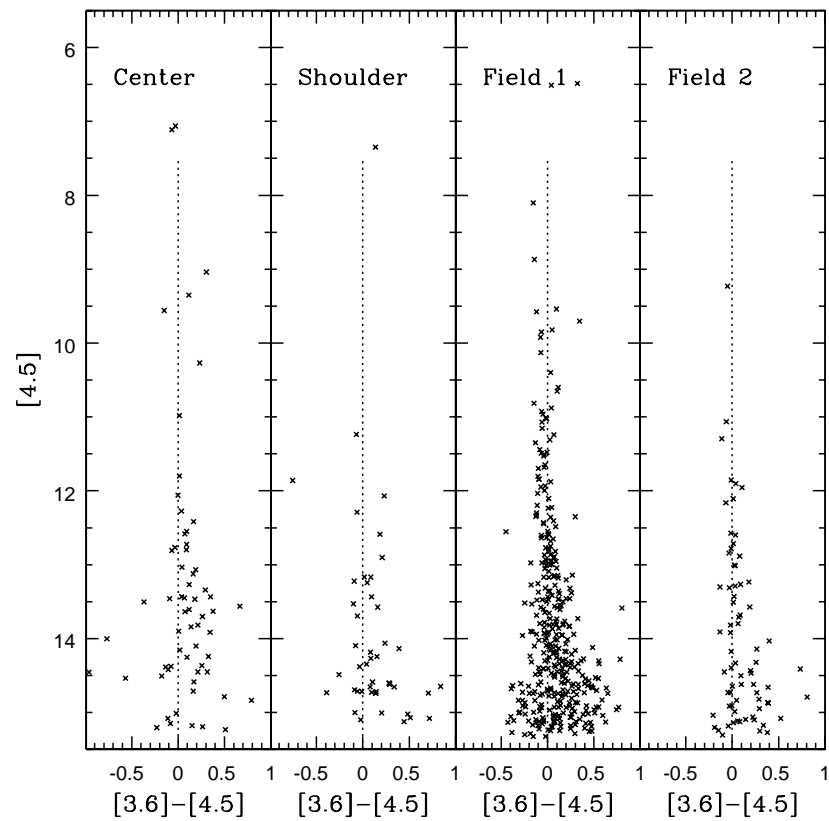

FIG. 7.- ([4.5], [3.6] - [4.5]) CMDs of NGC 3105 and the surrounding area. The dotted line in each panel indicates [3.6]-[4.5] $=0$, which is the approximate color of photospheric light. Some of the stars in the Center region with $i^{\prime}$ between 9 and 11 have redder [3.6]-[4.5] colors than the majority of objects, and this is likely due to emission from circumstellar material.

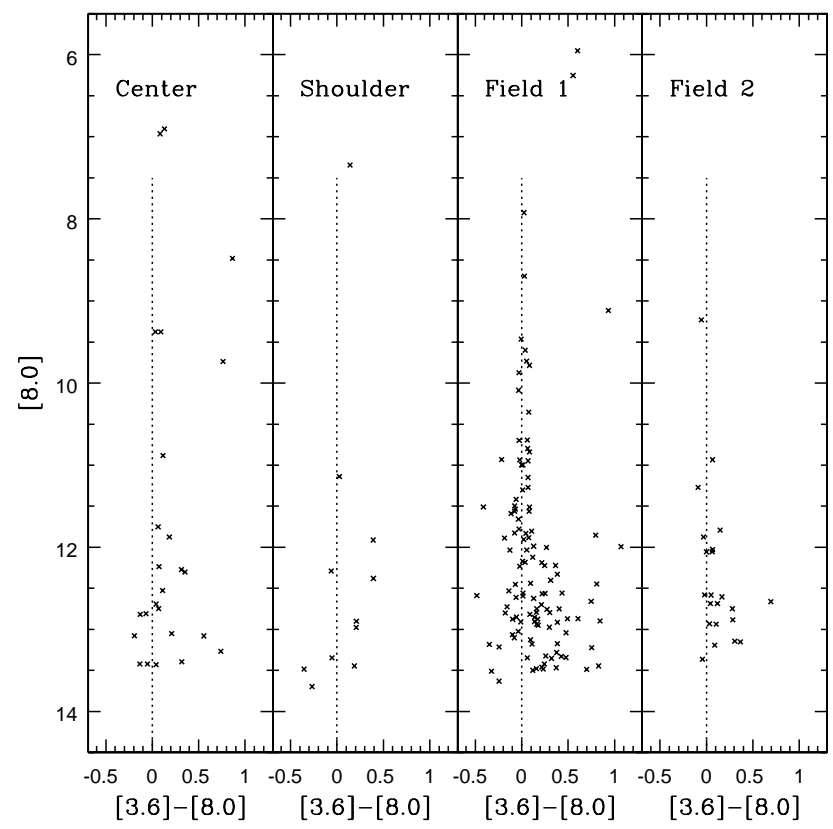

FIG. 8.- Same as Figure 7, but showing ([8.0], [3.6]-[8.0]) CMDs. The bright stars near the cluster center that have red [3.6]-[8.0] colors are the same ones that have red [3.6]-[4.5] colors in Figure 7 .

3105. The brightest of these is Star 7 in the compilations of Moffat \& Fitzgerald (1974) and FitzGerald et al. (1977), which they classify as a Be star. A modest number of sources with red [3.6]-[4.5] and [3.6]-[8.0] colors are also seen in Field 1, and in Section 7 it is shown that emission line stars are also detected in this part of the
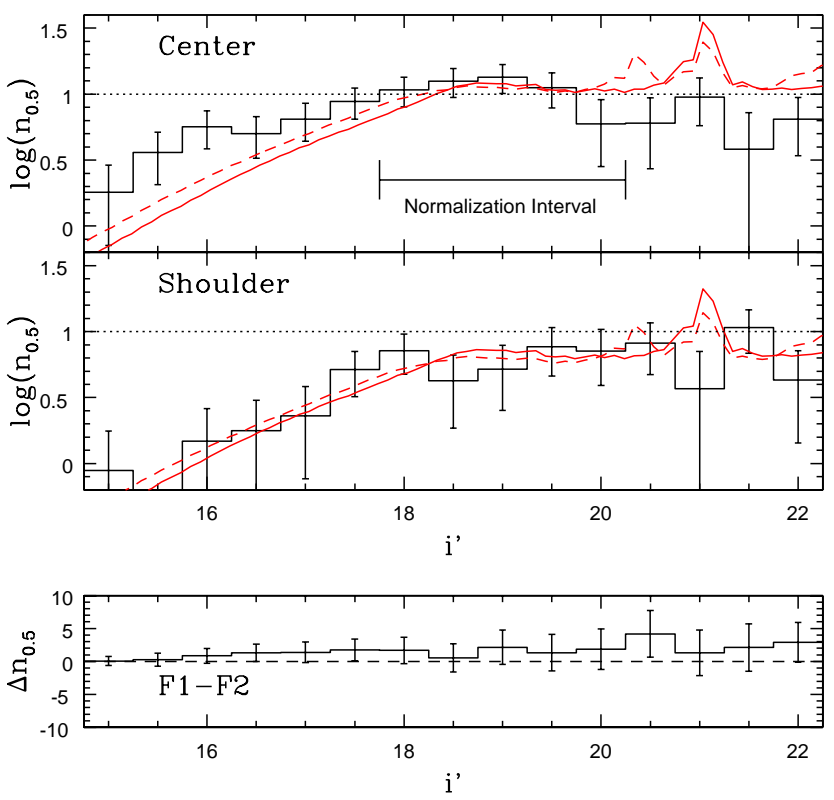

Fig. 9.- (Top two panels): $i^{\prime}$ LFs of the Center and Shoulder fields. $\mathrm{n}_{0.5}$ is the number of stars per square arcmin per 0.5 magnitude in $i^{\prime}$, corrected statistically for non-cluster objects by subtracting the Field 2 LF after scaling for differences in sky coverage. The error bars show counting statistics. The dotted line in each panel marks $\log \left(\mathrm{n}_{0.5}\right)=1$. While the number density of stars with $i^{\prime}>19.5$ is more-or-less similar in the two regions, the number of MS stars with $i^{\prime}<18$ clearly drops with increasing radius - the ratio of bright to faint stars in the Center region is higher than in the Shoulder region. The red line is a model LF generated from the Bressan et al. (2012) isochrones for single stars (i.e. no binaries), assuming an age of $32 \mathrm{Myr}, \mathrm{Z}=0.008$, and the distance modulus and $\mathrm{E}(\mathrm{B}-\mathrm{V})$ used in Figure 5. A Chabrier (2003) lognormal MF has been adopted, and the LF has been scaled to match the observations in the $i^{\prime}$ interval that is indicated. The dashed red line shows a model LF that includes equal mass binaries with a 0.5 binary fraction. (Lower panel:) Difference in number counts between Field 1 and Field 2. There is a systematic tendency for Field 1 to contain more objects than Field 2, suggesting that some stars associated with NGC 3105 may be present in Field 1. sky.

\section{THE LUMINOSITY FUNCTION AND SPATIAL DISTRIBUTION OF STARS}

Cluster LFs contain encoded information about the mass function (MF) of the component stars. The $i^{\prime}$ LFs of the Center and Shoulder regions are shown in Figure 9. Only stars that were detected in both filters were counted.

Stars associated with NGC 3105 may be present in Field 1, and evidence to support this is presented in the lower panel of Figure 9, where the difference between the Field 1 and $2 \mathrm{LFs}$ is shown. While there are no statistically significant differences between the two LFs in any one $i^{\prime}$ bin, the counts in Field 1 are systematically higher than those in Field 2 at all magnitudes, suggesting that objects associated with NGC 3105 are present in Field 1. A modest excess numbers of stars in Field 1 when compared with Field 2 is also consistent with the [3.6] surface brightness profile in Figure 2. Field 2 was thus adopted as the background field, and a statistical correction for field stars was applied to the LFs in Figure 9 by subtracting the Field 2 LF.

The projected density of objects with $i^{\prime} \geq 19.5$ is 
similar in the Center and Shoulder regions. When averaged over tens of arcsec, objects with $i^{\prime}$ between 19.5 and 22.0 are thus more-or-less uniformly distributed within 70 arcsec of the cluster center. In contrast, the number of stars with $i^{\prime} \leq 19$ in the Center region is $\sim 0.4-0.5$ dex (i.e. a factor of $\sim 3 \times$ ) higher than in the Shoulder region. These differences in the distribution of bright and faint stars point to mass segregation in NGC 3105.

To further explore the nature of any radial variation in stellar content, model LFs based on the $\mathrm{Z}=0.008$ Padova 32 Myr isochrone were constructed, and these are shown in Figure 9. The models assume a Chabrier (2003) lognormal mass function, and have been normalized to match the number counts in the magnitude interval indicated in the top panel. The distance modulus and reddening used in Figure 5 have been adopted for this comparison.

The solid line in Figure 9 shows a model LF that assumes no binaries, whereas the dashed line shows a model that includes equal mass binaries with a binary fraction 0.5, as estimated in Section 5. As expected, the model that includes binaries falls above the non-binary model at the bright end. A model LF that assumes the same binary fraction but a Chabrier mass distribution for companions (i.e. mass ratios different from unity) would fall midway between the equal mass and non-binary models.

The bump in the single star model LF in Figure 9 near $i^{\prime}=21$ is due to the MSCO, which can be used to estimate age if the distance and reddening of a young cluster are known. The amplitude of the MSCO is strongest in very young clusters, and weakens with increasing age. The MSCO will be difficult to detect in NGC 3105 given the statistical errors in the number counts, and there is no corresponding feature in the cluster LF near this magnitude.

The models in Figure 9 match the observations in the Shoulder region, suggesting that the isolated stars and dominant members of binary systems in that part of NGC 3105 can be characterized by a Chabrier MF, at least as faint as $i^{\prime}=22\left(\sim 0.7-0.8 \mathrm{M}_{\odot}\right)$. In contrast, there is a systematic excess in the Center region LF when compared with the models in the magnitude interval $i^{\prime}<18\left(>1.7 \mathrm{M}_{\odot}\right)$. The difference between the models and observations in the Center region would become larger yet if the models were normalized at fainter magnitudes than shown in Figure 9. The models indicate that binarity affects the number counts at the bright end of the LF by no more than $\sim 0.2 \mathrm{dex}$, and so it is unlikely that binarity can explain the central excess of bright objects.

\section{STELLAR SPECTRA}

The locations and photometric properties of stars for which spectra were obtained are listed in Table 3, while the $\left(i^{\prime}, g^{\prime}-i^{\prime}\right)$ CMD of these objects, with $\mathrm{Z}=0.008$ isochrones from Bressan et al. (2012) over-plotted, is shown in Figure 10. The targeted stars sample three narrow magnitude intervals to facilitate star-to-star comparisons at different points on the cluster CMD. Brightnessrelated biases are reduced because the stars in each group have comparable magnitudes. The two objects with unusually red colors near $i^{\prime} \sim 15.5$ and 18.5 were observed because they could be placed in a slit that contained a

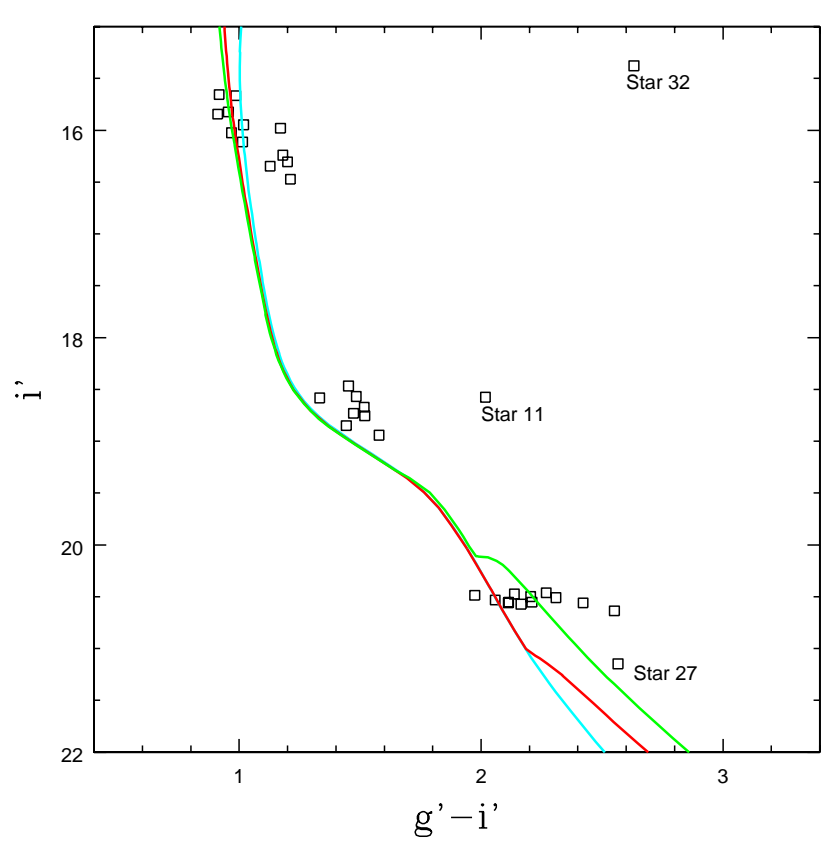

Fig. 10. - CMD of stars for which spectra were recorded. The target stars sample three parts of the CMD: (1) the upper MS (AB stars), (2) the middle part of the MS (A-F stars), and (3) the magnitude where PMS stars might be expected based on past age estimates (likely G dwarfs). Stars 11, 27, and 32 were observed because they could be placed in the same slit as a high priority target. $\mathrm{Z}=0.008$ isochrones for ages $20 \mathrm{Myr}$ (green), $32 \mathrm{Myr}$ (red), and $72 \mathrm{Myr}$ (cyan) are also shown.

primary spectroscopic target. The faintest star in the sample, with $i^{\prime} \sim 21.0$, was also observed for this reason.

Cluster members in the brightest group sample the upper MS. Members of NGC 3105 in this group will have $\mathrm{M}_{V} \sim 0$, and so are expected to have A - B spectral types. Based on previous spectroscopic studies of stars near NGC 3105 (Moffat \& FitzGerald 1974; FitzGerald et al. 1977), the spectra of some of these might contain emission features. Early-type stars are also of interest for assessing interstellar absorption as there are large wavelength intervals that are free of strong photospheric lines.

The stars with $\mathrm{M}_{i^{\prime}} \sim 18.5$ sample objects midway down the MS in NGC 3105 with $\mathrm{M}_{V} \sim 2.5$. Stars with this $\mathrm{M}_{V}$ have $\mathrm{A}-\mathrm{F}$ spectral-types, and these spectra may contain detectable metallic absorption lines. The faintest group, with $\mathrm{M}_{i^{\prime}} \sim 20.5$, samples stars in NGC 3105 that have $\mathrm{M}_{V} \sim 5.5$. Cluster members at the brightness will be $\mathrm{G}$ dwarfs if they are on the MS.

\subsection{The Bright Star Sample}

The bright sample can be sub-divided into two groups - one with $g^{\prime}-i^{\prime}<1.1$ that hugs the isochrone sequence in Figure 10, and a second that has redder colors. Spectra centered on $\mathrm{Na} \mathrm{D}$ and $\mathrm{H} \alpha$ of stars in the blue and red sub-samples are shown in Figures 11 and 12. The mask alignment stars, the spectra of which were recorded through a square aperture rather than a slit, are indicated with an asterisk. All three alignment stars are in the red sub-sample. None of the stars in the bright sample are in the Center or Shoulder regions, as primacy for spectroscopic targeting in those areas was assigned to fainter objects.

There are only modest star-to-star differences be- 


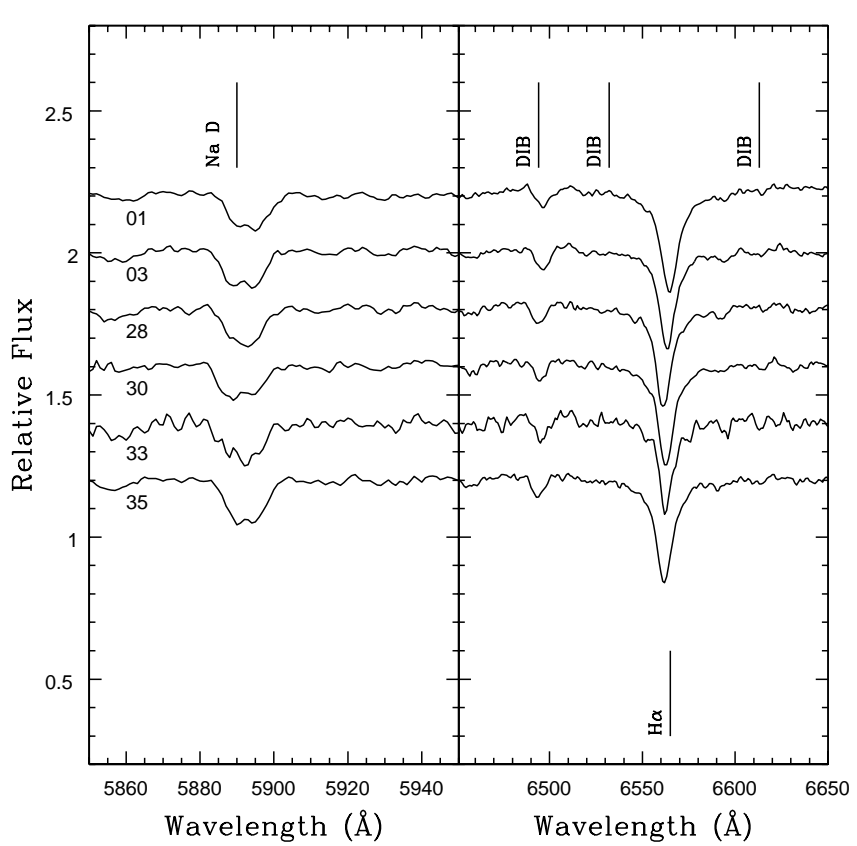

FIG. 11. - Spectra of stars in the bright sample that have $g^{\prime}-i^{\prime}<$ 1.1. Wavelengths centered near $\mathrm{Na} \mathrm{D}$ and $\mathrm{H} \alpha$ are shown. DIBs that have mean EWs > $0.1 \AA$ in the Diffuse Interstellar Band Catalogue (http://leonid.arc.nasa.gov/DIBcatalog.html) are indicated.

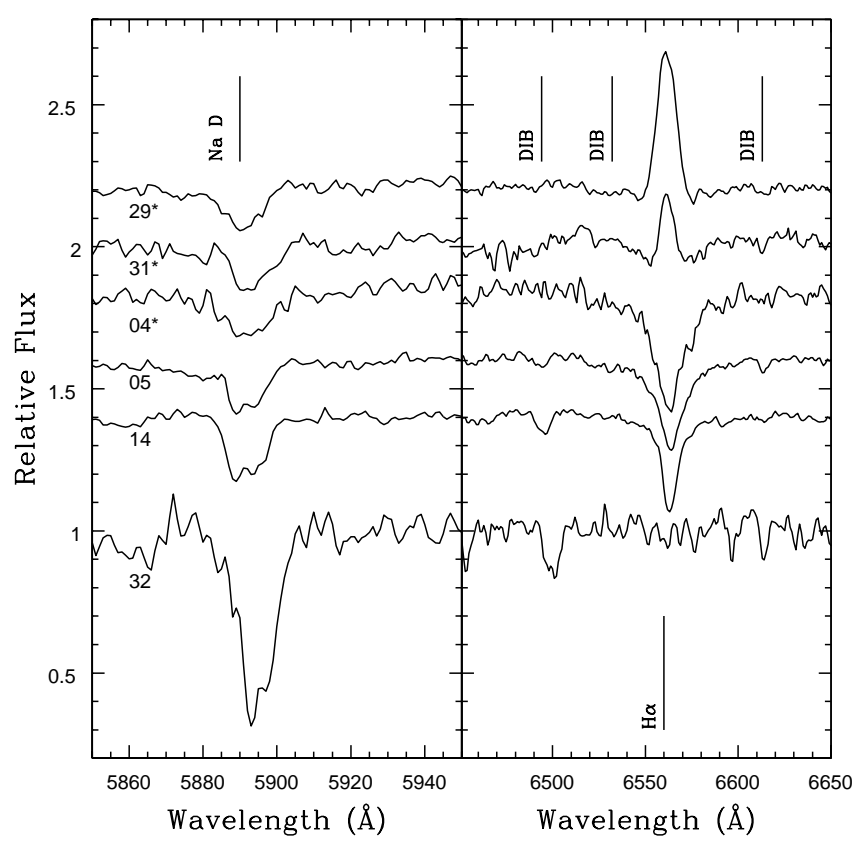

FIG. 12.- Same as Figure 11 but showing spectra of stars in the bright sample with $g^{\prime}-i^{\prime}>1.1$. Alignment stars are indicated with an asterick next to their identification \#. The stars with $\mathrm{H} \alpha$ emission are placed at the top for display purposes. There is noticeable star-to-star dispersion in the depths of $\mathrm{Na} D$ and the DIB at $6494 \AA$. Star 32 has spectroscopic properties that are very different from those of the other bright objects, and it is likely a foreground K dwarf (Section 7.3). tween the spectra in Figure 11. However, this is not the case among the spectra in Figure 12. Stars 29 and 31 are alignment stars, and have $\mathrm{H} \alpha$ in emission, suggesting that they are Be stars. Such stars are associated with systems that have ages up to a few tens of millions of years, and Fitzgerald et al. (1977) identify three Be stars within 5 arcmin of the center of NGC 3105. Star 29 in the current sample is likely star 78 in the Moffat \& FitzGerald (1974) study, although those authors did not assign a spectral type for that star. As alignment objects, Stars 29 and 31 were selected to have a location in the focal plane that did not interfere with the spectra of other objects - that the spectra of both show $\mathrm{H} \alpha$ in emission provides further evidence that Be stars are common in the area around NGC 3105. While Stars 29 and 31 have relatively red $g^{\prime}-i^{\prime}$ colors, the IRAC photometry indicates that neither shows excess IR emission at wavelengths $\leq 8 \mu \mathrm{m}$, although both are near the faint limit of the [8.0] GLIMPSE measurements. The absence of an IR excess would suggest that they are not of type B[e] (e.g. Allen \& Swings 1976).

Star 32, which has the reddest $g^{\prime}-i^{\prime}$ color in Figure 10 , has a spectrum that differs from that of the other members of the bright group. Na D in the Star 32 spectrum is much deeper than in the other spectra, and $\mathrm{H} \alpha$ is abscent. The EW of $\mathrm{Na} \mathrm{D}$ in the Star 32 spectrum is $6.5 \pm 0.7 \AA$, and this is consistent with that of a mid-K dwarf (e.g. Diaz et al. 2007). If Star 32 has a spectraltype K5V (Section 7.3) then $\left(g^{\prime}-i^{\prime}\right)_{0} \sim 1.2$ and so E $\left(g^{\prime}-\right.$ $\left.\mathrm{i}^{\prime}\right) \sim 1.4$. This corresponds to $\mathrm{E}(\mathrm{B}-\mathrm{V}) \sim 0.8$ using the $\mathrm{R}=3.1$ reddening law of Cardelli et al. (1989). This is marginally lower than the reddening of NGC 3105, and is consistent with Star 32 being in the foreground.

With the exception of Star 32, the stars in the bright sample have early spectral types. The $\mathrm{H} \alpha$ absorption line profiles tend to be sharper than expected from MS stars, and are similar to those in the spectra of evolved B stars. This is demonstrated in Figure 13, where the mean spectrum of the stars in the blue subsample is compared with spectra of B7V (HD 43153)and B7III (HD 35497) stars from the Le Borgne et al. (2003) library. Spectra in the Le Borgne et al. (2003) compilation have a resolution $\sim 2000$, and so the spectra have been smoothed with a Gaussian to match the resolution of the GMOS spectra. The $\mathrm{H} \alpha$ line profile in the B7V spectrum is clearly broader than in the B7III and mean GMOS spectra.

There is also significant interstellar Na D absorption in the spectra of stars in the bright sample. The spectrum of the O5e star HD46223 from the Le Borgne et al. (2003) compilation is shown in Figure 13. This star has a line-of-sight extinction $\mathrm{A}_{V} \sim 2.1$, and Na D absorption in its spectrum has a depth that is not greatly different from that of the stars in the bright sample. There are only modest star-to-star differences in the depth of $\mathrm{Na} \mathrm{D}$ in Figure 11, and this likely reflects the similar colors (and hence reddenings) of the stars in this sample. There is greater dispersion in the depth of $\mathrm{Na} \mathrm{D}$ among the stars in the red sample, with Star 14 showing the deepest $\mathrm{Na} \mathrm{D}$ feature among the bright early-type stars.

Star-to-star variations in the depth of the $6494 \AA$ DIB are evident in the right hand panel of Figure 12. This DIB is weakest in the two stars with $\mathrm{H} \alpha$ emission, 


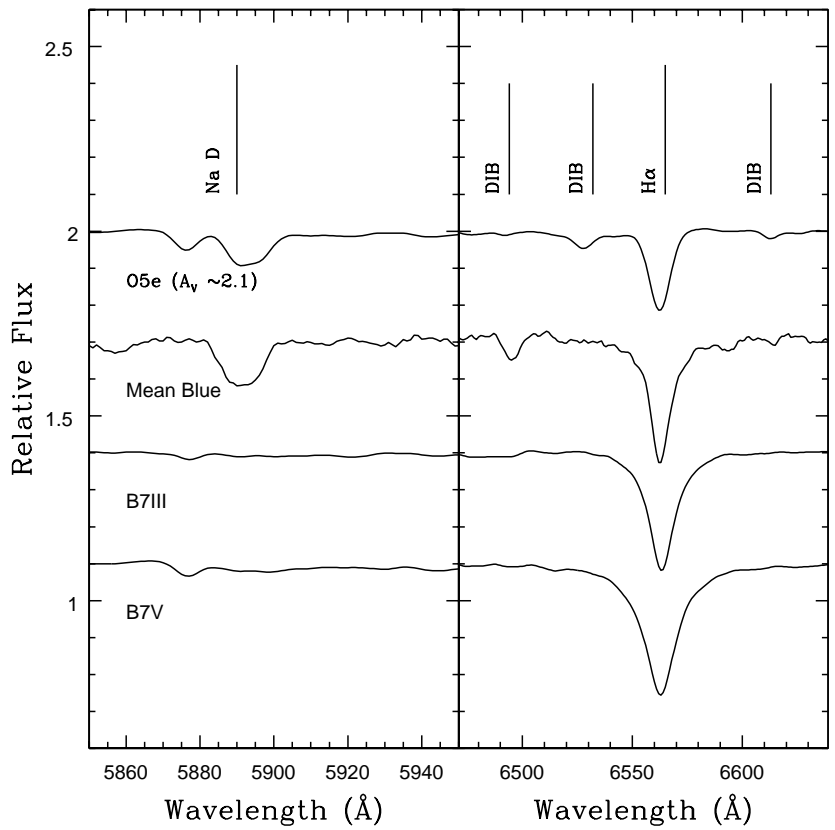

FIG. 13. - Mean spectrum of stars in the bright sample that have $g^{\prime}-i^{\prime}<1$.1. Spectra from the Le Borgne et al. (2003) library that have been smoothed to match the resolution of the GMOS spectra are also shown. $\mathrm{Na} \mathrm{D}$ is clearly visible in the spectrum of the O5e star HD 46223, which has a line-of-sight extinction $\mathrm{A}_{V} \sim 2.1$. Luminosity effects are explored with spectra of the B7III star HD 35497 and the B7V star HD 43153. The $\mathrm{H} \alpha$ line profile in the mean blue spectrum is more akin to that in $\mathrm{B}$ giants as opposed to $\mathrm{B}$ dwarfs.

but can be seen in the spectra of Stars 05 and 14. The depth of the 6494 DIB tends to correlate only weakly with those of other DIBs (Cami et al. 1997). Cami et al. (1997) also find a possible anti-correlation with the $6613 \AA$ feature, which is marked in the right hand panel of Figure 12.

Equivalent width (EW) measurements of $\mathrm{Na} \mathrm{D}$ were made from mean spectra that were constructed for three different groupings: (1) all of the blue sub-sample stars, (2) the mean spectrum of Stars 4, 5, and 14, which are the stars in the red sub-sample that do not show $\mathrm{H} \alpha$ emission and are not foreground dwarfs, and (3) the mean spectrum of all stars in the bright sample, but again excluding the $\mathrm{H} \alpha$ emission Stars 29 and 31 and the foreground dwarf Star 32. The results are listed in Table 4. The uncertainties in Table 4 reflect the dispersion in EWs found by perturbing the start and end points of the EW measurements.

TABLE 4

NA D EQUivalent WidThS (EWs)

IN MEAN SPECTRA CONSTRUCTED FROM THE BRIGHT SAMPLE.

\begin{tabular}{lc}
\hline ID & EW \\
$\#$ & $(\AA)$ \\
\hline Blue Sub-sample & $1.5 \pm 0.1$ \\
Stars 4, 5, \& 14 & $1.9 \pm 0.1$ \\
Bright Sample & $1.6 \pm 0.1$ \\
(No Stars 29, 31, \& 32) & \\
\hline
\end{tabular}

The depths of the Na D lines in the spectra of early type stars can be used to estimate their reddening.

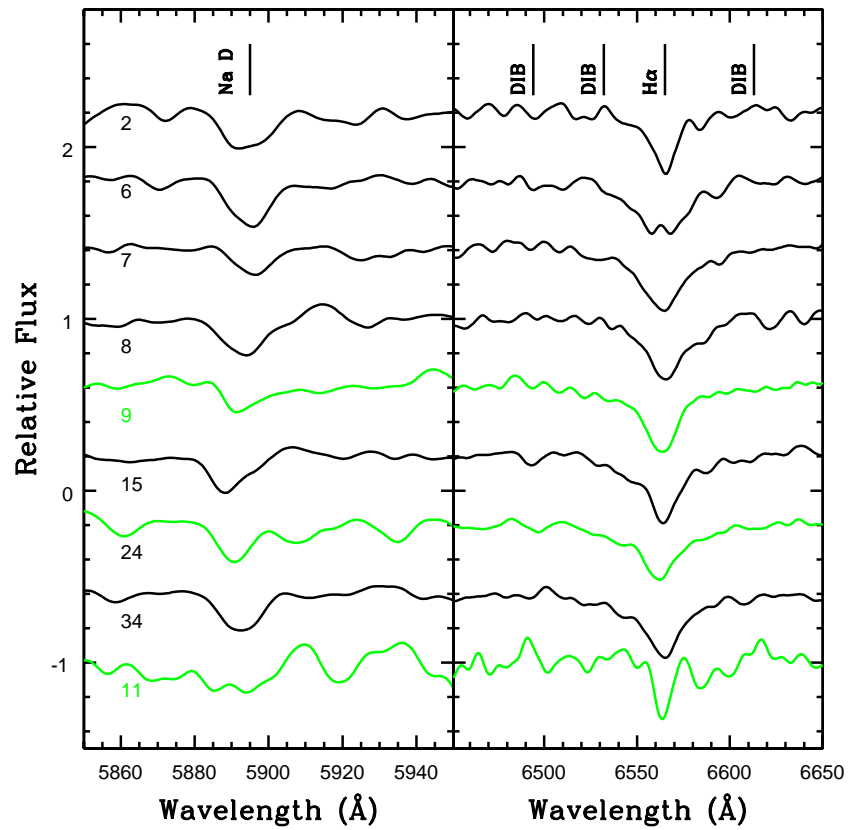

FIG. 14.- Spectra near the $\mathrm{Na} \mathrm{D}$ and $\mathrm{H} \alpha$ lines of intermediate brightness stars. The spectra shown in black are of stars in Fields 1 and 2, while the green spectra are of stars in the Shoulder region. The spectra have been smoothed to a $9.5 \AA$ resolution. The spectrum of Star 11, which has a red color and was observed because it could be placed in the same slit as a higher priority target, is shown at the bottom of the figure. The majority of these stars show broad $\mathrm{H} \alpha$ absorption that is characteristic of A stars. The locations of the DIBs marked in Figure 11 are shown.

Poznanski et al. (2012) find a relation between the EW of the $\mathrm{Na} \mathrm{D}$ lines and $\mathrm{E}(\mathrm{B}-\mathrm{V})$. A blind application of equation 9 of Poznanski et al. (2012) to the Na D EW measured for the early-type stars in the blue sub-sample yields $\mathrm{E}(\mathrm{B}-\mathrm{V})=0.80 \pm 0.25$. Applying the $\mathrm{Na} \mathrm{D} \mathrm{EW}$ from the entire bright sample using the entry in the last row of Table 4 yields $\mathrm{E}(\mathrm{B}-\mathrm{V})=1.05 \pm 0.25$. These values agree with the reddening measured from the CMD. There is thus consistency between the $g^{\prime}-i^{\prime}$ color of the MS and the depth of the $\mathrm{Na} \mathrm{D}$ feature in the spectra of early-type stars in the NGC 3105 field.

\subsection{Intermediate Magnitude Stars}

Spectra centered on $\mathrm{Na} \mathrm{D}$ and $\mathrm{H} \alpha$ of stars in the intermediate brightness sample are compared in Figure 14. The spectra have been smoothed to a resolution of $9.5 \AA$ to boost the $\mathrm{S} / \mathrm{N}$ ratio while maintaining a spectral resolution that is sufficient to allow strong absorption features to be identified (e.g. Worthey et al. 1994; Pickles 1998). Star 11, which is displayed at the bottom of the figure, was observed because it could be placed in the same slit as a higher priority target.

There is a range of $\mathrm{H} \alpha$ characteristics in Figure 14. Star 6 has $\mathrm{H} \alpha$ in emission, while Star 11 has a narrow $\mathrm{H} \alpha$ profile. The other stars have broad $\mathrm{H} \alpha$ profiles that are reminiscent of A spectral-types. Despite smoothing, considerable noise remains in the spectra near $\mathrm{Na} \mathrm{D}$, with Star 11 having the lowest S/N ratio near $5900 \AA$ because of its very red color. Star 6 has the deepest Na D absorption. Circumstellar material may contribute to Na D absorption in the spectrum of this star given the presence of $\mathrm{H} \alpha$ emission in its spectrum. 


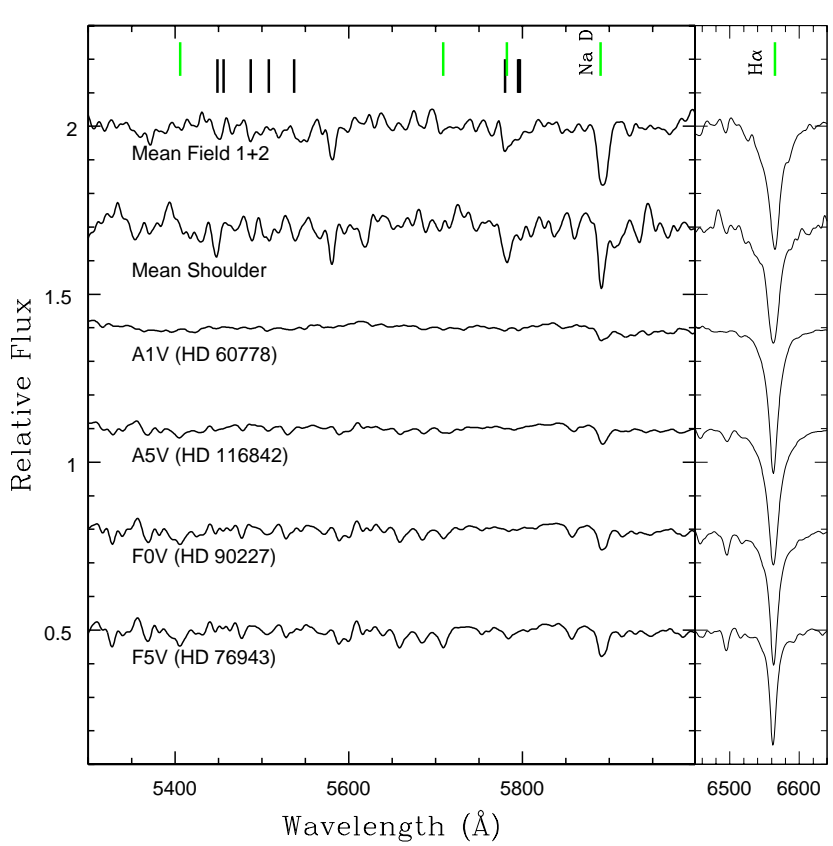

FIG. 15. - Mean spectra of intermediate magnitude stars in Field $1+2$ and the Shoulder regions. The spectra have been smoothed to a spectral resolution of $9.5 \AA$. Spectra of stars from the Le Borgne et al. (2003) library that have been normalized to the continuum and smoothed to match the resolution of the GMOS spectra are also shown. DIBs that have mean EWs $>0.1 \AA$ in the Diffuse Interstellar Band Catalogue (http://leonid.arc.nasa.gov/DIBcatalog.html) are indicated, and the green lines mark the locations of Fe absorption features at 5335, 5406, 5709, and 5782A. Efforts to estimate spectral-types at wavelengths $<6000 \AA$ in these spectra are complicated by noise and interstellar absorption features. However, the depth and shape of $\mathrm{H} \alpha$ absorption in the Shoulder and Field spectra is consistent with a mid-A spectral type.

The spectra of stars 9 and 24, which are in the Shoulder region, were combined, as were the spectra of Stars 2, 7, 8, 15, and 34-Star 6 was not considered when computing the Shoulder region mean given the presence of $\mathrm{H} \alpha$ emission. Stars from the Le Borgne et al. (2003) library are compared with the combined GMOS spectra in Figure 15. The reference spectra have been smoothed to the same resolution as the GMOS data. The wavelength interval shown in Figure 15 contains wellcalibrated probes of metallicity and surface gravity (e.g. Worthey et al. 1994).

The Na D line in both mean spectra is deeper than in the bright star sample, suggesting that there is a photospheric contribution to $\mathrm{Na} \mathrm{D}$ absorption in the intermediate magnitude sample. Efforts to classify the mean GMOS spectra are complicated by noise and interstellar absorption features at wavelengths $<6000 \AA$, and so we rely on $\mathrm{H} \alpha$ to estimate spectral-types. The depth and shape of $\mathrm{H} \alpha$ in the median cluster and field spectra are consistent with a spectral type $\sim \mathrm{A} 5 \mathrm{~V}$. This was confirmed by subtracting reference spectra from the mean GMOS spectra and examining the residuals.

A distance modulus can be computed if it is assumed that the stars in the intermediate brightness sample follow the solar-neighborhood $\mathrm{M}_{V}$ vs. spectral-type relation. Jaschek \& Gomez (1998) examine the $\mathrm{M}_{V}$ calibration of spectroscopic standards, and their Table 1 suggest that $\mathrm{M}_{V}=1.7 \pm 0.3$ for an $\mathrm{A} 5 \mathrm{~V}$ star. The uncer- tainty reflects the difference in $\mathrm{M}_{V}$ for the two $\mathrm{A} 5 \mathrm{~V}$ stars in the Jaschek \& Gomez sample. The stars in the intermediate magnitude sample have $i^{\prime} \sim 18.7$, and if $\mathrm{E}(\mathrm{B}-\mathrm{V})$ $=1$ this then corresponds to $V \sim 19.3$, or $V_{0} \sim 16.2$. The distance modulus of the star in the intermediate sample is then $\sim 14.5 \pm 0.3$. This agrees with the values found from the CMDs in Section 4. While it is not clear if the stars in the intermediate sample belong to NGC 3105, they appear to be at a similar distance.

\subsection{Faint Stars}

If the stars in the faint sample are on the MS of NGC 3105 then the isochrones suggest that they should have a $\mathrm{G}$ spectral-type. It is difficult to estimate the spectral-types of individual stars in the faint sample as the spectra have a low $\mathrm{S} / \mathrm{N}$ ratio. However, $\mathrm{Na} \mathrm{D}$ is a deep absorption feature in the spectrum of late-type dwarfs, and it is seen in many of the faint sample spectra. Na D provides a means of identifying foreground dwarfs, as these objects will have lower masses than stars in NGC 3105, and so their spectra will have deeper Na D absorption.

Even though the majority of objects in the faint sample were intentionally selected to be in the Cluster or Shoulder regions to boost the probability of cluster membership, source counts suggest that roughly half of the stars in the faint sample will not be cluster members (Figure 6). In fact, four stars in the faint sample (\#'s 10, 19, 25, and 26) were found to have Na D absorption that was much deeper than that in the other objects, and these were flagged as candidate field stars. A median spectrum of the stars in the faint sample, but excluding \#'s 10, 19, 25, and 26, was constructed. The median was taken in an effort to suppress the influence of any field stars that might remain in the sample. The resulting spectrum, smoothed to a resolution of 400 to boost the $\mathrm{S} / \mathrm{N}$ ratio, is shown in Figure 16. Spectra of $\mathrm{G}$ and $\mathrm{K}$ stars from the Le Borgne et al. (2003) compilation, smoothed to the same resolution, are also shown in that figure.

$\mathrm{H} \alpha$ emission might be expected if there was largescale chromospheric activity among the stars in the faint group, and this is not seen in the median spectrum. Rather, there appears to be weak $\mathrm{H} \alpha$ absorption in the combined spectrum. As for $\mathrm{Na} \mathrm{D}$, this feature has an EW of $\sim 7.8 \AA$ in the median faint sample spectrum. After accounting for interstellar absorption, $\mathrm{Na} \mathrm{D}$ in the median spectrum is much deeper than expected in G dwarfs, and is consistent with an early to mid-K spectral-type.

There are interstellar absorption features in the median spectrum that complicate spectral classification based on comparisons with stars that are subject to only modest levels of extinction. As a late-type foreground dwarf that is also heavily extincted, Star 32 is thus an interesting comparison object for the faint sample median spectrum. The spectrum of Star 32, smoothed to a resolution of 400, is shown in Figure 16. There are many similarities between the median faint sample spectrum and that of Star 32. The lack of $\mathrm{H} \alpha$ absorption in the Star 32 spectrum suggests that it has a later spectral-type than the majority of the faint sample members. Based on the comparisons in Figure 16 it appears that Star 32 has a spectral-type in the range $\mathrm{K} 5 \mathrm{~V}-\mathrm{K} 7 \mathrm{~V}$.

The deep $\mathrm{Na} \mathrm{D}$ and weak $\mathrm{H} \alpha$ absorption in the 


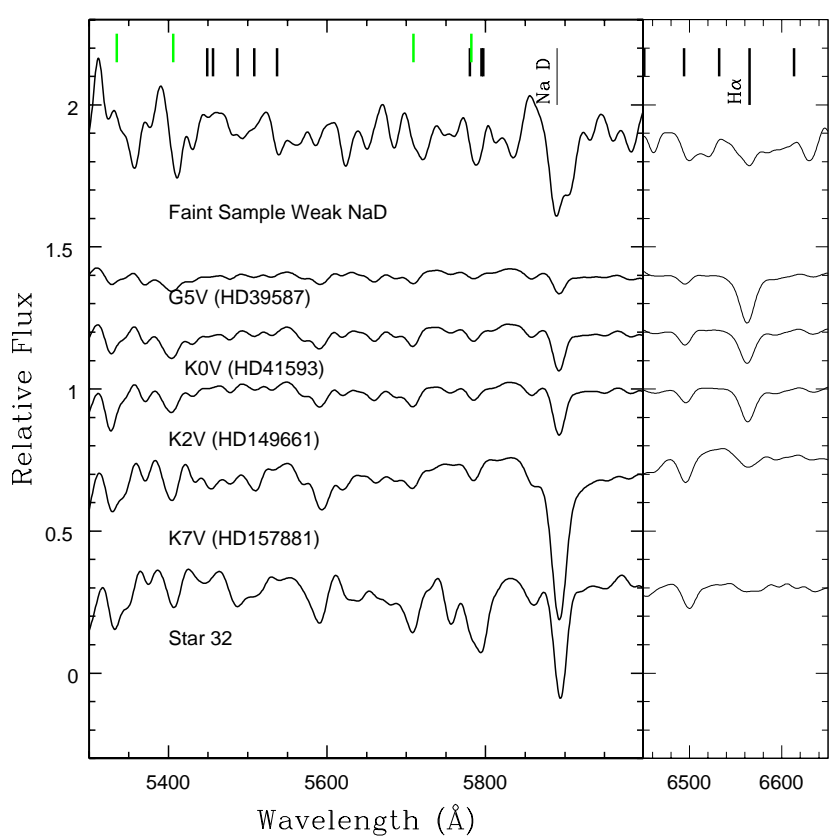

FIG. 16.- Median spectrum of stars in the faint sample - stars that have strong $\mathrm{Na} \mathrm{D}$ absorption, and thus are suspected foreground dwarfs, have been excluded. Also shown are spectra of $\mathrm{G}$ and $\mathrm{K}$ stars from the Le Borgne et al. compilation, and the smoothed spectrum of Star 32. All spectra have a resolution $\lambda / \Delta \lambda=400$. The locations of strong DIBs and Fe absorption lines from Figure 15 are also indicated. The depths of $\mathrm{Na} \mathrm{D}$ and $\mathrm{H} \alpha$ suggests a $\mathrm{K}$ spectral type for the majority of stars that make up the faint star sample. Star 32 from the bright sample likely has a spectral type in the range $\mathrm{K} 5 \mathrm{~V}-\mathrm{K} 7 \mathrm{~V}$, and its spectrum has features in common with some of those in the median faint sample spectrum.

median faint sample spectrum is perhaps surprising given that a G spectral type was expected for these stars. Star spot activity has the potential to affect the effective temperature of stars if the spots fall over a large part of the stellar disk. Spotted single stars will have lower effective temperatures than unspotted models, and models examined by Somers \& Pinsonneault (2015) suggest that the impact on effective temperature is $\sim 200-300 \mathrm{~K}$. This is not sufficient to produce the difference between the observed and expected spectral types. Large-scale spot activity might also produce chromospheric emission, and no evidence for this is seen. A more pragmatic explanation is that - the culling of obvious field stars based on $\mathrm{Na} \mathrm{D}$ depth notwithstanding - foreground stars dominate the sample. Spectra of stars with colors and magnitudes like those in the faint sample with a higher $\mathrm{S} / \mathrm{N}$ ratio will be useful to distinguish between cluster and field stars at this brightness in NGC 3105.

\section{SUMMARY \& DISCUSSION}

Images and spectra obtained with GMOS on GS have been used to investigate the age, distance, and reddening of the outer Galactic disk open cluster NGC 3105. The $g^{\prime}$ and $i^{\prime}$ GMOS images allow photometry to be obtained of sources that are much fainter than in previous studies at visible/red wavelengths, reaching magnitudes where cluster PMS stars might be expected if NGC 3105 has an age $\sim 20 \mathrm{Myr}$, as suggested by some age estimates. The spectra enable the first detailed reconnaisance of the stellar content of NGC 3105 and the surrounding field below the MSTO, and yield independent reddening and distance estimates.

There is good agreement between the reddening and distance found from the photometry and spectra. The reddening estimated from the locus of MS stars on the CMD agrees with that found from the depth of $\mathrm{Na}$ $\mathrm{D}$ absorption in the spectra of bright early-type stars in and around NGC 3105 using a calibration found by Poznanski et al. (2012). The distance estimated from MS-fitting is consistent with that found in previous studies, and also agrees with the distance estimated from the spectral-types of candidate MS stars at intermediate magnitudes. We note that these stars are located throughout the GMOS science field, and so are not restricted to the dense inner regions of the cluster where membership probabilities are high.

An age of at least $32 \mathrm{Myr}$ is estimated for NGC 3105 based on the photometric properties of (1) the brightest stars near the cluster center, which include MSTO and post-MS objects, and (2) the morphology of the lower parts of the CMD. An upper limit to the age range is $71 \mathrm{Myr}$, and this is defined by the photometric properties of candidate upper MS stars. The isochrones suggest that the GMOS observations do not sample PMS stars in NGC 3105, and so deeper observations will be required to find these objects at visible/red wavelengths. This may prove to be challenging as it can be difficult to identify stars with sub-solar masses in low latitude clusters as foreground/background stars may have similar photometric properties. In the case of very young clusters individual PMS stars that are surrounded by accretion discs may be identified based on their distinct photometric and spectroscopic properties. However, the circumstellar discs that produce these signatures have a characteristic decay time of a few Myr (Fedele et al. 2010, but see also e.g. De Marchi et al. 2011a), negating their usefulness as beacons for identifying sources in clusters as old as NGC 3105. Still, Scicluna et al. (2014) suggest that in some cases circumstellar discs may be rejuvenated by the accretion of fresh molecular material, potentially increasing the time interval over which disks may be detected. In any event, ensembles of candidate stars can be identified statistically by examining the spatial distribution and photometric properties of objects (e.g. Davidge 2015 ), as is done here.

The consistency of age estimates based on observations that span a range of wavelengths are an important test of stellar structure models. Davidge (2014) used near-infrared observations to examine the CMD and LF of NGC 3105 in the sub-solar mass regime. The $(K, J-K) \mathrm{CMD}$ of the central $85 \times 85$ arcsec of NGC 3105 constructed by Davidge (2014) shows a well-defined sequence at faint magnitudes that was attributed to the PMS. The PMS sequence in the $(K, J-K) \mathrm{CMD}$ is bracketed by 20 and $40 \mathrm{Myr}$ isochrones from Bressan et al. (2012). The age of NGC 3105 estimated from the PMS locus in that CMD is 25-30 Myr. Considering only the faintest stars in the GMOS CMD, the comparisons in Figure 6 suggest that NGC 3105 has an age $>22$ Myr. The ages derived from PMS/faint MS stars in the near-IR and at visible/red wavelengths are thus consistent.

Davidge (2014) also examined the $K$ LF of the center of NGC 3105 to a faint limit $K \sim 19.5$, which 
corresponds to $\sim 0.5 \mathrm{M}_{\odot}$. The flat shape of the $K \mathrm{LF}$ at the faint end is similar to the $i^{\prime} \mathrm{LF}$, which is flat from $i^{\prime} \sim 18\left(\sim 3.5 \mathrm{M}_{\odot}\right)$ to $i^{\prime} \sim 22\left(\sim 0.8 \mathrm{M}_{\odot}\right)$, and is matched by models that have a Chabrier (2003) MF. However, Davidge (2014) found that model LFs that are based on the Bressan et al. (2012) isochrones and that assume a solar neighborhood $\mathrm{MF}$ do not reproduce the flat nature of the $K \mathrm{LF}$ at faint magnitudes. The $K$ observations probe less massive stars than the GMOS $i^{\prime}$ data. If the interpretation of the $K \mathrm{LF}$ is correct then a downturn in the $i^{\prime}$ LF will occur $1-2$ magnitudes below the faint limit of the GMOS data.

There is excess MIR emission associated with some of the brightest stars in the GMOS science field, as expected if these objects have circumstellar disks. The spectra of four of the stars in the bright and intermediate brightness samples have $\mathrm{H} \alpha$ in emission, which is not surprising as line emission is common among earlytype stars in systems with ages $>10 \mathrm{Myr}$. If these stars are not cluster members then they may have formed more-or-less concurrently with NGC 3105 in structures that have dispersed into the field. Simulations of star formation in molecular clouds discussed by Bonnell et al. (2011) suggest that both clustered and diffuse components form. While many young star-forming regions will expand and dissipate, some retain primordial substructures (e.g. Gregorio-Hetem et al. 2015), and NGC
3105 may be such a remnant. Diffusely distributed young populations might be expected to survive longer in the low density regions of the outer Galactic disk than in higher density areas at smaller Galactocentric radii.

The presence of a diffuse bright population notwithstanding, star counts indicate that the brightest, most massive members of NGC 3105 are more centrally concentrated than the fainter, low mass members, indicating that there is mass segregation. The central concentration of bright members might have a primordial origin. Simulations (e.g. Dale et al. 2015) suggest that - in the absence of feedback - star formation rates in forming clusters tend to be highest in regions where the potential well is deepest. In fact, evidence for primordial segregation is seen in the Orion star-forming region (e.g. Hillenbrand \& Hartmann 1998), and simulations suggest that it can also occur in very low mass clusters (Kirk et al. 2014). While feedback can suppress star formation in dense regions (e.g. Dale et al. 2015), clusters that have a low stellar mass will have lower probablities of forming the massive stars that drive feedback than more massive systems, and so long-lived central knots may form preferentially in low mass clusters. There is an observational bias favoring the detection of low mass clusters that have bright, central concentrations, and so the observed frequency of such systems may overestimate their actual numbers.

\section{REFERENCES}

Adams, F. C., Hollenbach, D., Laughlin, G., \& Gorti, U. 2004, ApJ, 611,360

Allen, D. A., \& Swings, J. P. 1976, A\&A, 47, 293

Banerjee, S., \& Kroupa, P. 2015, MNRAS, 447, 728

Bell, C. P. M., Naylor, T., Mayne, N. J., Jeffries, R. D., \& Littlefair, S. P. 2012, MNRAS, 424, 3178

Benjamin, R. A., Churchwell, E., Babler, B. L., et al. 2003, PASP, 115,953

Bik, A., Stolte, A., Gennaro, M., et al. 2014, A\&A, 561, A12

Bonnell, I. A., Smith, R. J., Clark, P. C., \& Bate, M. R. 2011, MNRAS, 410, 2339

Bressan, A., Marigo, P., Girardi, L. et al. 2012, MNRAS, 427, 127

Cami, J., Sonnentrucker, P., Ehrenfreund, P., \& Foing, B. H. 2007, A\&A, 326, 822

Cardelli, J. A., Clayton, G. C., \& Mathis, J. S. 1989, ApJ, 345, 245 Chabrier, G. 2003, PASP, 115, 763

Cignoni, M., Tosi, M., Nota, A., et al. 2010, ApJ, 712, L63

Dale, J. E., Ercolano, B., \& Bonnell, I. A. 2015, MNRAS, 451, 987

Davidge, T. J. 2014, ApJ, 781, A95

Davidge, T. J. 2015, PASP, 127, 836

de Grijs, R., Goodwin, S. P., \& Anders, P. 2013, MNRAS, 436, 136

De Marchi, G., Francesco, P., Panagia, N., et al. 2011a, ApJ, 739, A27

De Marchi, G., Panagia, N., \& Sabbi, E. 2011b, ApJ, 740, A10

Fall, S. M., \& Chandar, R. 2012, ApJ, 752, 96

Farias, J. P., Smith, R., Fellhauer, M., Goodwin, S., Candlish, G. N., Blana, M., \& Domunguez, R. 2015, MNRAS, 450, 2451

Fazio, G. G., Hora, J. L., Allen, L. E., et al. 2004, ApJS, 154, 10

Fedele, D., van den Ancker, M. E., Henning, T., Jayawardhana, R., \& Oliveira, J. M. 2010, A\&A, 510, A72

Fitzgerald, M. P., Jackson, P. D., \& Moffat, A. F. J. 1977, A\&A, 59, 141

Getman, K. V., Feigelson, E. D., Kuhn, M. A., et al. 2014, ApJ, 787,108

Gregorio-Hetem, J., Hetem, A., Santos-Silva, T., \& Fernandes, B. 2015, MNRAS, 448, 2504

Hillenbrand, L. A., \& Hartmann, L. W. 1998, ApJ, 492, 540

Hollyhead, K., Bastian, N., Adamo, A., Silva-Villa, E., Dale, J., \& Royon, J. E. 2015, MNRAS, 449, 1106

Hook, I. M., Jorgensen, I., Allington-Smith, J. R., Davies, R. L., Metcalfe, N., Murowinski, R. G., \& Crampton, D. 2004, PASP, 116,425
Jaschek, C., \& Gomez, A. E. 1998, A\&A, 330, 619

Johnstone, D., Hollenbach, D., \& Bally, J. 1998, ApJ, 499, 758

Kirk, H., Offner, S. S. R., \& Redmond, K. J. 2014, MNRAS, 439, 1765

Kruijssen, J. M. D., Maschberger, T., Moeckel, N., et al. 2012, MNRAS, 419, 841

Kudryavtseva, N., Brandner, W., Gennaro, M., et al. 2012, ApJ, $750, \mathrm{~L} 44$

Kuhn, M. A., Getman, K. V., \& Feigelson, E. D. 2015, ApJ, 802, 60

Lada, C. J., \& Lada, E. A. 2003, ARA\&A, 41, 57

Le Borgne, J.-F., Bruzual, G., Pello, R., et al. 2003, A\&A, 402, 433

Lim, B., Chun, M-Y, Sung, H., et al. 2013, AJ, 145, 46

Lyra, W., Moitinho, A., van der Bliek, N. S., \& Alves, J. 2006, A\&A, 453, 101

Messineo, M., Clark, J. S., Figer, D. J., et al. 2015, ApJ, 805, 110

Moffat, A. F. J., \& Fitzgerald, M. P. 1974, A\&AS, 16, 25

Nakamura, F., \& Li, Z-Y 2014, ApJ, 783, 115

Palous, J., Wunsch, R., \& Tenorio-Tagle, G. 2014, ApJ, 792, 105

Parker, R. J., \& Meyer, M. R. 2014, MNRAS, 442, 3722

Paunzen, E., Netopli, M., Iliev, I. K., Maitzen, H. M., Claret, A., \& Pintado, O. I. 2005, A\&A, 443, 157

Pfalzner, S., Parmentier, G., Steinhausen, M., Vincke, K., \& Menten, K. 2014, ApJ, 794, 147

Pfalzner, S., Vincke, K., \& Xiang, M. 2015, A\&A, 576, A28

Pickles, A. J. 1998, PASP, 110, 863

Poznanski, D., Prochaska, J. X., \& Bloom, J. S. 2012, MNRAS, 426,1465

Reach, W. T., Megeath, S. T., Cohen, M., et al. 2005, PASP, 117, 978

Román-Zúñiga, C. G., Ybarra, J. E., Megías, G. D., Tapia, M., Lada, E. A., \& Alves, J. F. 2015, AJ, 150, 80

Sagar, R., Munari, U., \& de Boer, K. S. 2001, MNRAS, 327, 23

Scicluna, P., Rosotti, G., Dale, J. E., \& Testi, L. 2014, A\&A, 566, L3

Siess, L., Dufour, E., \& Forestini, M. 2000, A\&A, 358, 593

Silva-Villa, E., \& Larsen, S. S. 2011, A\&A, 529, A25

Silva-Villa, E., Adamo, A., Bastian, N., Fouesneau, M., \& Zackrisson, E. 2014, MNRAS, 440, L116

Stetson, P. B. 1987, PASP, 99, 191

Stetson, P. B., \& Harris, W. E. 1988, AJ, 96, 909 
Werner, M. W., Roellig, T. L., Low, F. J., et al. 2004, ApJS, 154, 1

Worthy, G., Faber, S. M., Gonzalez, J. J., \& Burstein, D. 1994, ApJS, 94, 687
Wright, E. L., Eisenhardt, P. R. M., Mainzer, A. K., et al. 2010, AJ, 140, 1868

Zeidler, P., Sabbi, E., Nota, A., et al. 2015, AJ, 150, 78 